The Free Internet Journal

for Organic Chemistry
Paper

Arkivoc 2017, part v, 172-186

\title{
Synthesis of some novel oxazolopyranoquinolinones from 3-amino-4-hydroxypyrano[3,2-c]quinolinedione
}

\author{
Hany M. Hassanin, ${ }^{* a}$ Ibrahim M. Abdou, ${ }^{b}$ and Abdeltawab M. Saeed ${ }^{a, b}$ \\ ${ }^{a}$ Department of Chemistry, Faculty of Education, Ain Shams University Roxy 11711 Cairo, Egypt \\ ${ }^{b}$ Department of Chemistry, College of Science, UAE University Al-Ain 15551, UAE \\ Email: hanyhassnin@yahoo.com
}

Received 05-29-2017

Accepted 07-04-2017

Published on line 10-08-2017

\section{Abstract}

6- $n$-Butyl-3-nitropyrano[3,2-c]quinolinone was chemoselectively reduced to an amine derivative in a good yield (70\%) using an efficient and simple tin-hydrochloric acid reaction. The novel 3-aminopyranoquinolinone was utilized as a precursor to produce some new interesting tetracyclic fused oxazolopyranoquinolinone analogues. The structures of the obtained quinolinone derivatives were confirmed using IR, LC-MS, ESI-MS and NMR techniques.

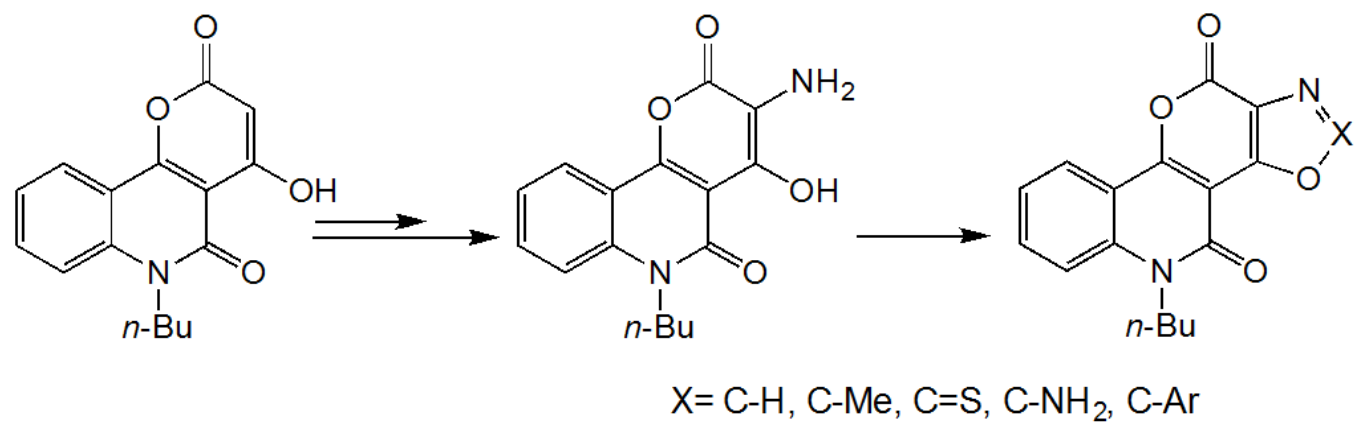

Keywords: Tin reduction, oxazole, pyran, quinolinone, heterocyclization 


\section{Introduction}

Pyranoquinoline skeletons including both a quinoline ring and a pyran moiety, afford exceptional biological activities. ${ }^{1-4}$ Rings annulated to the pyranoquinolinone units showed potential medicinal properties such as antibacterial, ${ }^{5}$ anticoagulant, ${ }^{6}$ antitumor, ${ }^{7}$ and microtubule-targeting agents. ${ }^{8}$ Despite the extensive body of published work on the synthesis of this type of compounds, we are not aware of any report that describes a simple procedure for the annulation of an oxazole nucleus to the pyranoquinolinone moiety to give a tetracyclic system. Oxazole derivatives have been found in numerous natural products and many of them have been shown to have an interesting broad range of biological activities, ${ }^{9,10}$ such as in inhibition of Streptococcus mutans biofilm formation, ${ }^{11}$ antipsychotics, ${ }^{12}$ HIV inhibitory, ${ }^{13}$ and anti-tumoral activities. ${ }^{14}$ It is also known that incorporation of fluorine atoms into molecules of heterocyclic compounds leads to a significant increase in their biological activities. ${ }^{15}$ Recently, the synthesis of fluoroquinolones has become of increasing interest since the quinoline skeleton is present in many chemotherapeutic agents. ${ }^{16-18}$ These findings prompted us to explore the combination of an oxazole nucleus and fluorine atom within the pyranoquinolinone moiety in one molecular framework. In continuation of our research focused on the chemistry of pyrano[3,2c]quinolinedione derivatives, ${ }^{19,20}$ we now disclose the preparation of a new synthetically valuable 3aminopyranoquinolone derivative in order to obtain a novel series of pyranoquinolinones incorporating an oxazole and/or fluorophenyloxazole at face $c$. We hope that these new compounds will have useful biological activities.

\section{Results and Discussion}

The nitration of 1 using a mixture of concentrated nitric acid and sulfuric acid gave 3-nitropyrano[3,2c]quinoline-2,5-dione 2. The ESI-MS of compound 2 showed four intense peaks at $\mathrm{m} / \mathrm{z} 331.1835,353.1434$, 354.2070 and 386.7095 , corresponding to $[\mathrm{M}+\mathrm{H}]^{+},[\mathrm{M}+\mathrm{Na}]^{+},[\mathrm{M}+\mathrm{Na}+\mathrm{H}]^{2+}$ and $[2 \mathrm{M}+\mathrm{Na}]^{+}$, respectively. After installing a nitro group in the 3-position of pyrano[3,2-c]quinoline-2,5-dione, we turned to its conversion into an amino group by an efficient chemoselective reduction method. Attempts to reduce the nitro compound 2 using activated iron ${ }^{21}$ or $\mathrm{FeCl}_{3} / \mathrm{Zn} / \mathrm{DMF} / \mathrm{H}_{2} \mathrm{O},{ }^{22}$ were not effective; a mixture of products was isolated, which could not be separated in a satisfactory manner to obtain any considerable amount of the amino derivative. Instead, we used tin, as previously described by Jampilek ${ }^{23}$ in the synthesis of 4hydroxy-3-nitroquinolin-2-one. Accordingly, the nitro-derivative $\mathbf{2}$ was reduced with tin and concentrated hydrochloric acid at $130{ }^{\circ} \mathrm{C}$ to produce 3-amino pyrano[3,2-c]quinoline-2,5-dione $\mathbf{3}$ in $70 \%$ yield (Scheme 1). The reduction proceeded efficiently with excellent chemoselectivity without affecting other functional carbonyl groups or the pyranoquinolinone system.

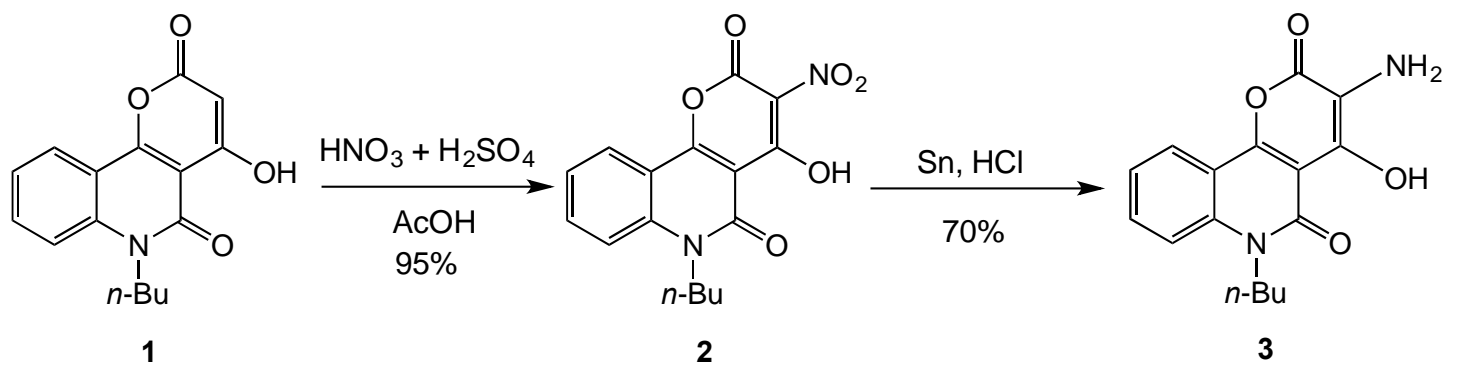

Scheme 1 
The formation of the amino analogue was confirmed by its IR spectrum showing bands at 3483 and 3395 $\mathrm{cm}^{-1}$ as a double peak due to the $\mathrm{NH}_{2}$ group. The mass spectrum of compound 3 revealed a molecular ion peak at $\mathrm{m} / \mathrm{z} 300$, corresponding to its formula weight (300.11). Moreover, compound $\mathbf{3}$ showed a quasimolecular ion peak at $\mathrm{m} / z 301.2[\mathrm{M}+\mathrm{H}]^{+}$and a sodiated molecular ion peak at $m / z 323.1[\mathrm{M}+\mathrm{Na}]^{+}$in the positive electron spray ionization-MS corresponding to $\mathrm{C}_{16} \mathrm{H}_{16} \mathrm{~N}_{2} \mathrm{O}_{4}$. There were two singlet signals in the gCOZY spectrum of compound 3: $3.72 \mathrm{ppm}$ characteristic of an $\mathrm{NH}_{2}$ group and $12.31 \mathrm{ppm}$ characteristic of the $\mathrm{OH}$ group (figure 1).<smiles>CCCCn1c(=O)c2c(O)c(N)c(=O)oc2c2c([18OH])c([125I])c([18OH])cc21</smiles>

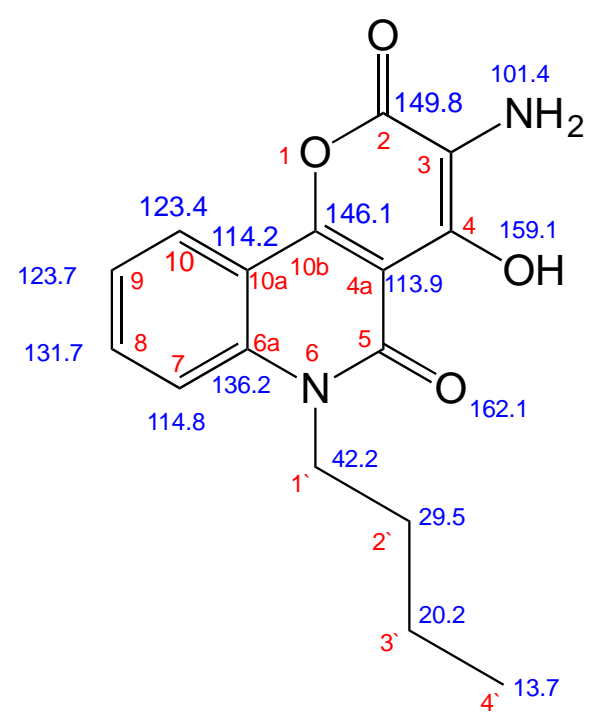

Figure $1 .{ }^{1} \mathrm{H}$ chemical shifts and correlation in COZY spectrum of compound 3.

The gHSQC, gHMBC and ${ }^{1} \mathrm{H},{ }^{13} \mathrm{C}-\mathrm{HMBC}$ spectra lead to assignment of the ${ }^{13} \mathrm{C}-$ chemical shift of compound 3 as shown in Figure 2.
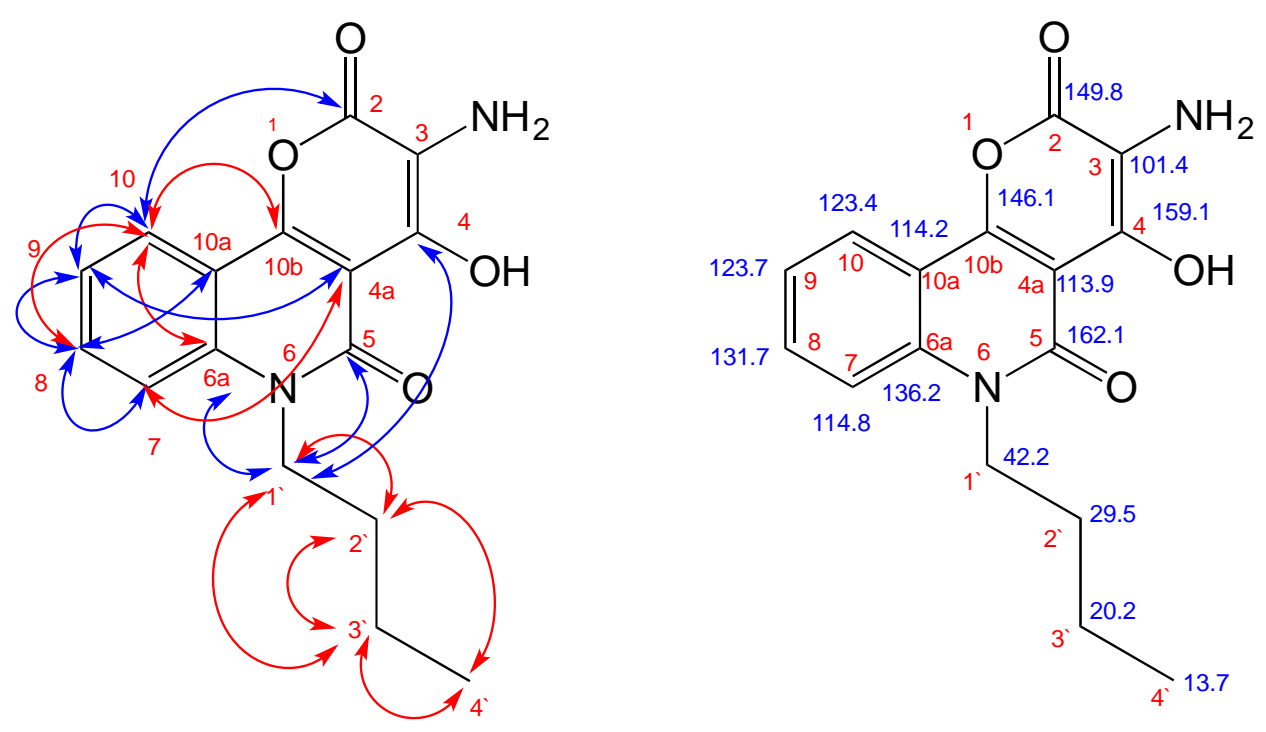

Figure 2. The network of $\mathrm{H}$-C long-range heteronuclear correlations and ${ }^{13} \mathrm{C}$ chemical shift assignment of compound 3. 
Heterocyclization at face $[c]$ of the pyrone moiety occurred when compound $\mathbf{3}$ was mixed with triethyl orthoformate, under solvent-free conditions. The reaction probably proceeds initially via condensation to produce the intermediate 4 . This intermediate can undergo intramolecular cyclondensation to give the desired oxazolopyrano[3,2-c]quinoline-4,5-dione 5 in low yield (42\%) (Scheme 2). The IR spectrum of compound 5 revealed the absence of bands due to the amino group. The ${ }^{1} \mathrm{H}$ NMR spectrum of compound 5 showed a new characteristic singlet signal at $8.79 \mathrm{ppm}$ characteristic of the $\mathrm{CH}$ of the oxazole ring. The spectrum also revealed the absence of deuterium-exchangeable protons, observed in the starting material as singlet signals, at 3.72 and $12.31 \mathrm{ppm}$, for the $\mathrm{N}-\mathrm{H}$ and $\mathrm{O}-\mathrm{H}$ protons, respectively. ${ }^{13} \mathrm{C}-\mathrm{NMR}$ spectrum of compound 5 demonstrated the presence of thirteen $s p^{2}$-hybridised carbons in the region 100-165 ppm due to the aromatic tetracyclic system. The same compound was obtained from the reaction of the amine $\mathbf{3}$ with formic acid in moderate yield $54 \%$.

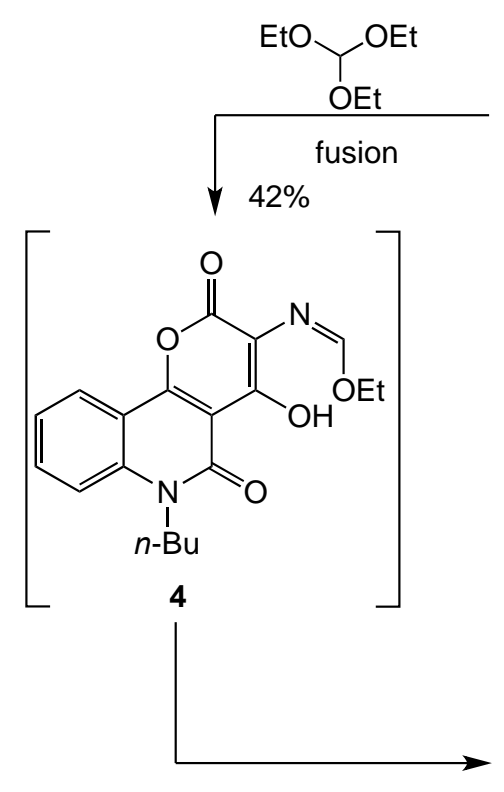<smiles>CCCCn1c(=O)c2c(O)c(N)c(=O)oc2c2ccccc21</smiles>

3

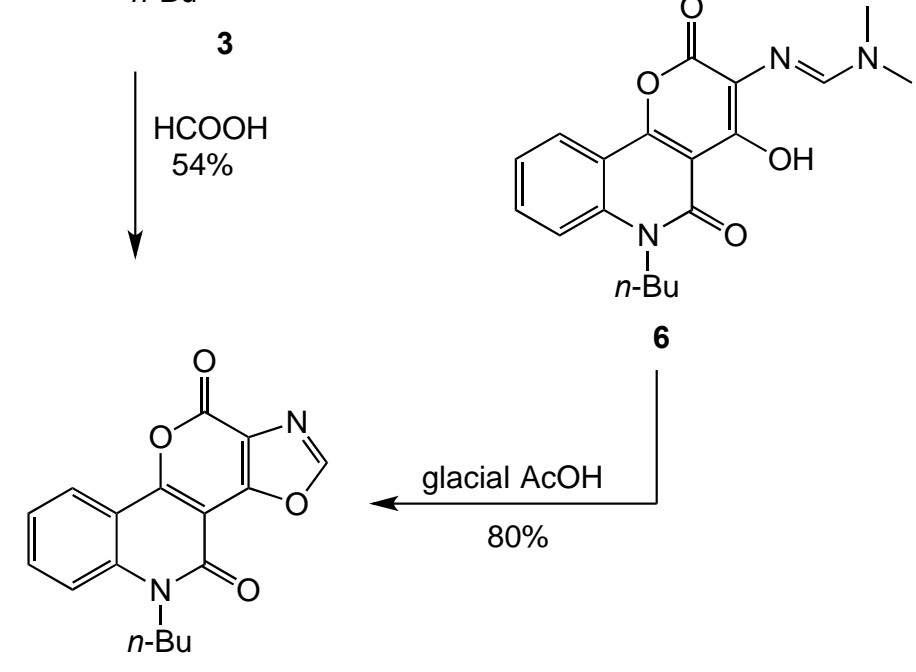

5

\section{Scheme 2}

Our attempts to improve the yield of the above reaction either by changing the reaction conditions or isolating the intermediate 4 were not successful. We expected that an alternative method for the preparation of compound 5 through a two-step reaction could produce better results. Thus, the intermediate enamine 6 was prepared by treating amine $\mathbf{3}$ with dimethylformamide dimethylacetal (DMF-DMA), and isolated by chromatography using ethyl acetate/hexane $6: 4$ as the eluent. The IR spectrum of enamine 6 revealed the absence of the double stretching bands of the amino group, as well as an ion peak at $\mathrm{m} / z 356.2$ in an ESI-MS $[\mathrm{M}+\mathrm{H}]^{+}$measurement in accordance with its structure. The ${ }^{1} \mathrm{H}$ NMR spectrum of the product showed two separate singlet signals at $3.05 \mathrm{ppm}$ and $3.14 \mathrm{ppm}$ attributed to two methyl protons of the $\mathrm{Me}_{2} \mathrm{~N}$ group, while the proton of the $(\mathrm{CH}=\mathrm{N})$ group was observed at $8.16 \mathrm{ppm}$. In the ${ }^{13} \mathrm{C}$ NMR spectrum of enamine 6 , two new $s p^{3}$ methyl carbons appeared at $\delta 31.1$ and 34.9, and a signal at $207.0 \mathrm{ppm}$ assignable to the $s p^{2}$ carbon of the 
$(\mathrm{CH}=\mathrm{N})$ group. Boiling enamine 6, in glacial acetic acid, (Scheme 2), effected its heterocyclization to afford oxazolopyran 5 in $80 \%$ yield. The time, yield and purity showed there are considerable advantages to the twostep sequence.

Homologue $\mathbf{8}$ was obtained by thermal cyclocondensation of the amine $\mathbf{3}$ with triethyl orthoacetate. As expected, this conversion occurred without isolation of porposed intermediate 7, in a low yield (46\%) (Scheme 3). Refluxing the amine $\mathbf{3}$ with acetic anhydride afforded the same oxazole derivative $\mathbf{8}$ in a somewhat greater yield, 52\%. Therefore, we adopted another procedure to synthesize compound $\mathbf{8}$, using a two-step synthesis. The first step was acetylation of the amine $\mathbf{3}$ to produce acetamide $\mathbf{9}$ as an isolated intermediate. The ESI-MS analysis of compound 9 showed an $[\mathrm{M}+\mathrm{H}]^{+}$ion at $\mathrm{m} / \mathrm{z} 343.1$, and an abundant $[\mathrm{M}+\mathrm{Na}]^{+}$ion at $\mathrm{m} / \mathrm{z}$ 365.1. The IR spectrum of acetyl derivative 9 had an absorption broad band characteristic for $(\mathrm{N}-\mathrm{H})$ at $3082 \mathrm{~cm}^{-1}$ and a new stretching band at $1703 \mathrm{~cm}^{-1}$ corresponding to the acetamide carbonyl group. In the ${ }^{1} \mathrm{H}$ NMR spectrum of 9, the protons of the acetyl group were observed at $2.06 \mathrm{ppm}$ as a singlet signal, and the $(\mathrm{N}-\mathrm{H})$ proton appeared at $9.12 \mathrm{ppm}$ as a singlet signal. The ${ }^{13} \mathrm{C}$ NMR spectrum of 9 exhibited the presence of two new signals at $23.2 \mathrm{ppm}$ and $207.0 \mathrm{ppm}$ due to the $\mathrm{CH}_{3}$ and $\mathrm{C}=\mathrm{O}$ of the acetyl group. Compound 9 was heated in refluxing $\mathrm{AcOH}$ and $\mathrm{Ac}_{2} \mathrm{O}$ to afford oxazole derivative 8 (Scheme 3). The disappearance of both hydroxyl group and carbonyl of the acetyl group are the prominent features in the IR spectrum of oxazole derivative 8 and its ESI-MS spectrum showed an $[\mathrm{M}+\mathrm{H}]^{+}$ion at $\mathrm{m} / \mathrm{z} 325.3$ and a $[\mathrm{M}+\mathrm{Na}]^{+}$ ion at 347.1. A [2M+Na] ${ }^{+}$ion was also observed at $m / z$ 671.1.

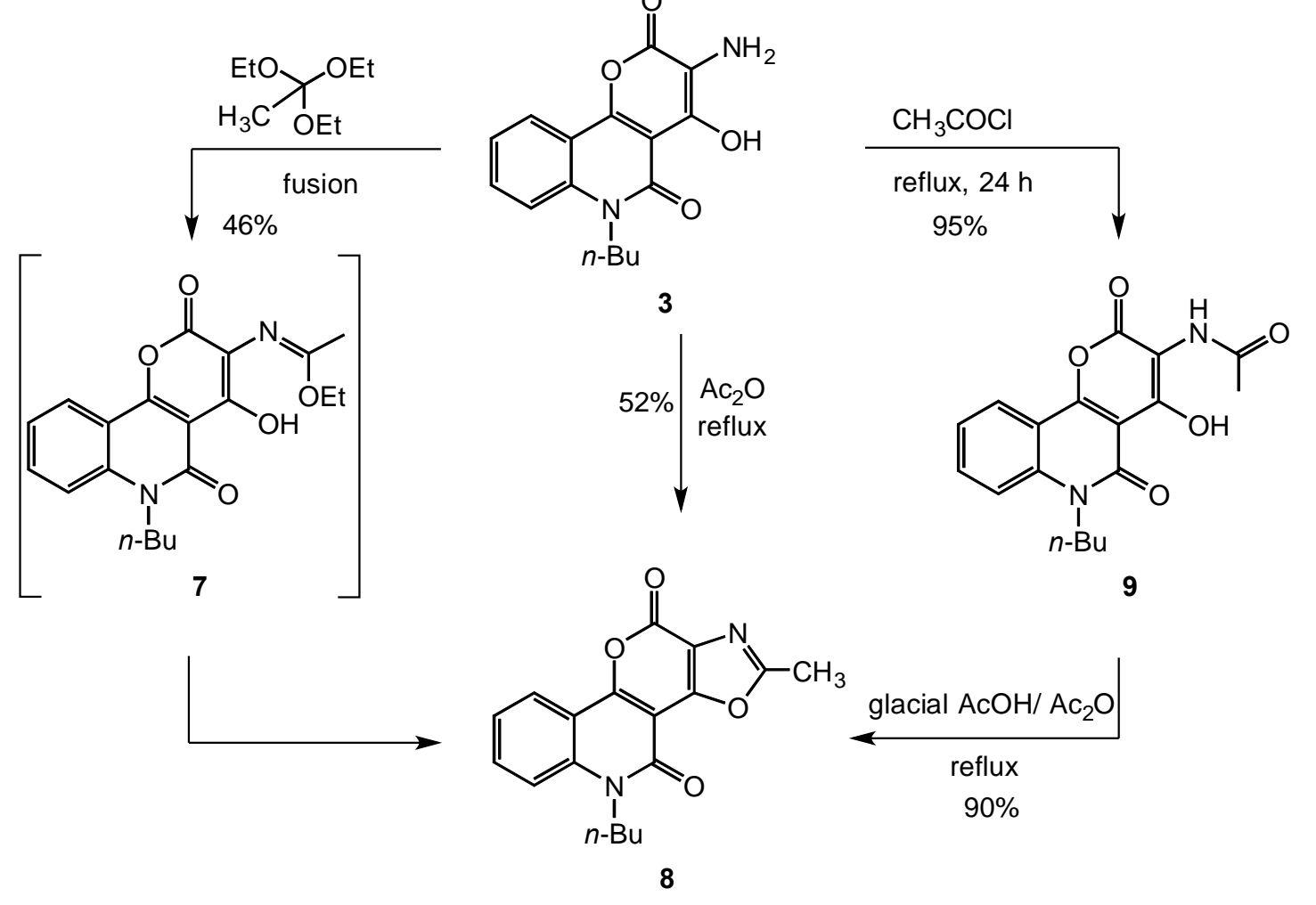

\section{Scheme 3}


The ${ }^{1} \mathrm{H}$ NMR signal for the methyl group on the oxazole ring was observed as a singlet at $2.73 \mathrm{ppm}$, while the $(\mathrm{N}-\mathrm{H})$ proton was no longer present. The ${ }^{13} \mathrm{C}-\mathrm{NMR}$ spectrum of compound 8 showed a signal at $13.8 \mathrm{ppm}$ for the methyl carbon atom at position 2 of the oxazole ring.

A nucleophilic addition reaction occurred when 3 was heated with phenyl isothiocyanate in absolute ethanol at reflux, producing phenylthiourea derivative $\mathbf{1 0}$ in a good yield (Scheme 4). The IR spectrum of compound 10 displayed characteristic absorption bands at 3342, $3156 \mathrm{~cm}^{-1}$ for NH groups and in the region of $1283 \mathrm{~cm}^{-1}$, corresponding to $\mathrm{C}=\mathrm{S}$ vibrations, in addition to an a-pyran carbonyl group at $1748 \mathrm{~cm}^{-1}$. The ${ }^{1} \mathrm{H}-$ NMR spectrum of 10 contained two singlet signals attributed to two NH protons, at 8.94 and 9.76 ppm. Signals integrating for the nine aromatic protons of the phenyl and benzo groups were observed in the region 7.06$8.19 \mathrm{ppm}$, confirming the formation of structure 10. There were two deuterium-exchangeable signals for the two $(\mathrm{N}-\mathrm{H})$ protons. The ${ }^{13} \mathrm{C}-\mathrm{NMR}$ of compound $\mathbf{1 0}$ had nineteen signals in the region $100-164$ ppm belonging to the aromatic carbon atoms and the thiocarbonyl group. The mass spectrum exhibited a molecular ion peak at $m / z 435(15 \%)\left(\mathrm{M}^{+}\right)$, along with $(\mathrm{M}+1)$ at $436(5 \%)$. The base peak at $\mathrm{m} / \mathrm{z} 189(100 \%)$ correspond to a molecular formula of $\mathrm{C}_{11} \mathrm{H}_{11} \mathrm{NO}_{2}$.

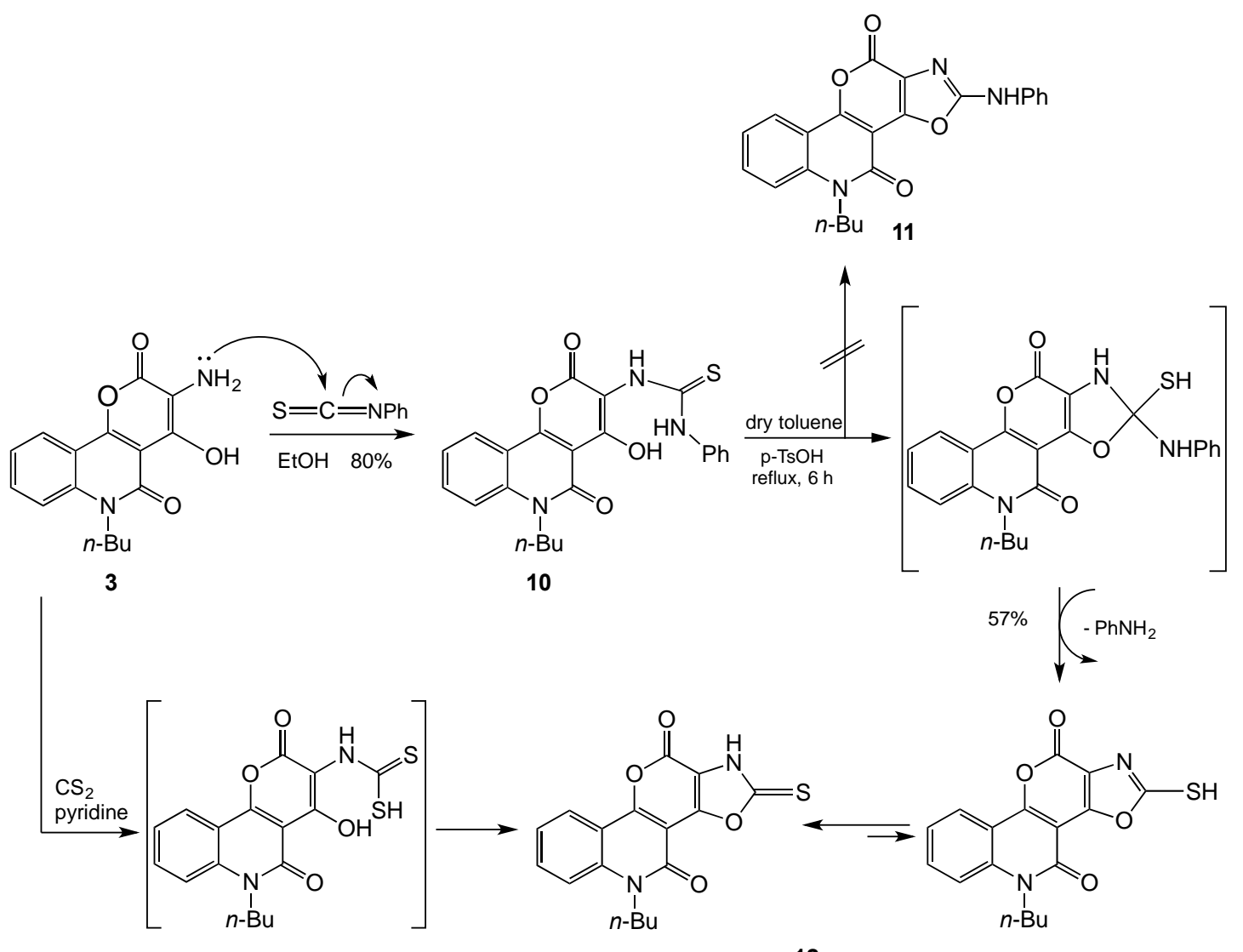

12

\section{Scheme 4}

We speculated that boiling phenylthiourea derivative $\mathbf{1 0}$ in dry toluene containing $p$-toluenesulfonic acid as a catalyst, would effect its intramolecular heterocyclization. The probable products are either oxazole 11 or oxazole 12. The IR spectrum of the product displayed characteristic absorption bands at 3342 and $3100 \mathrm{~cm}^{-1}$ for $\mathrm{NH}$, in addition to a-pyrano carbonyl group at $1745 \mathrm{~cm}^{-1}$. The ${ }^{1} \mathrm{H}-\mathrm{NMR}$ spectrum of the product was characterized by the presence of only four aromatic signals in the region $7.36-8.05$ ppm corresponding to the 
protons of the benzne ring of the quinolinone. There was, in addition, a deuterium-exchangeable singlet signal assignable to an $\mathrm{NH}$ proton at 10.78 . The ${ }^{13} \mathrm{C}-\mathrm{NMR}$ spectrum had a signal at $177 \mathrm{ppm}$ due to $\mathrm{C}=\mathrm{S}_{\text {oxazole, }}$ and thirteen other signals belonging to the aromatic carbon atoms of the tetracyclic system. The mass spectrum of the product showed a molecular ion peak at $\mathrm{m} / z 342\left(\mathrm{M}^{+}\right)(50 \%)$ corresponding to a molecular formula of $\mathrm{C}_{17} \mathrm{H}_{14} \mathrm{~N}_{2} \mathrm{O}_{4} \mathrm{~S}$. Building on the above data, the structure is confirmed as oxazolopyranoquinolinone 12 . The proposed pathway for the formation of compound $\mathbf{1 2}$ is thought to involve an intramolecular additionelimination as depicted in Scheme 4. We suggest that an initial nucleophilic addition of the phenolic group to the thiocarbonyl function of the neighboring side-chain is followed by loss a molecule of aniline. The structure of compound $\mathbf{1 2}$ was chemically confirmed via its direct preparation from the reaction of $\mathbf{3}$ and carbon disulfide in boiling pyridine.

In another approach towards the nucleophilic addition followed by cyclization to give an oxazolo pyranoquinolinone derivative, was reacting compound $\mathbf{3}$ with ammonium thiocyanate in boiling pyridine (Scheme 5). Lassaigne's test and elemental microanalysis of the product showed the absence of sulfur, so the product cannot contain sulfur. The IR spectrum revealed strong absorption bands at 3488 and $3394 \mathrm{~cm}^{-1} \mathrm{due}$ to stretching vibration of $\mathrm{NH}_{2}$.

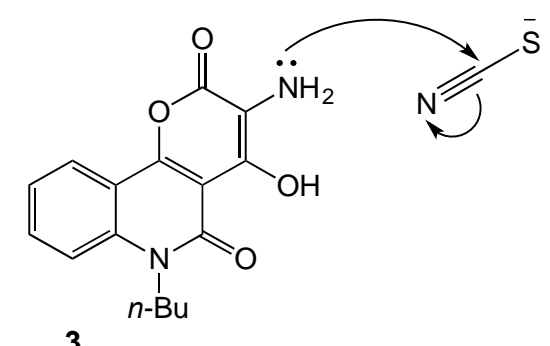

3

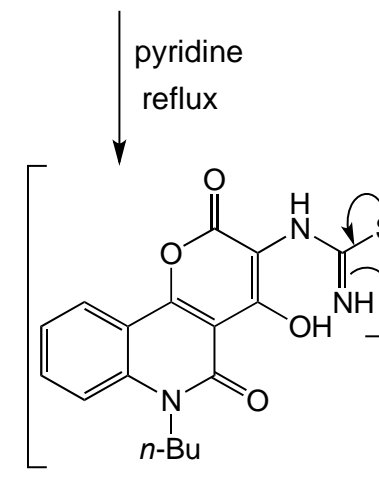<smiles>CCCCn1c(=O)c2c3oc(N)nc3c(=O)oc2c2ccccc21</smiles>

13
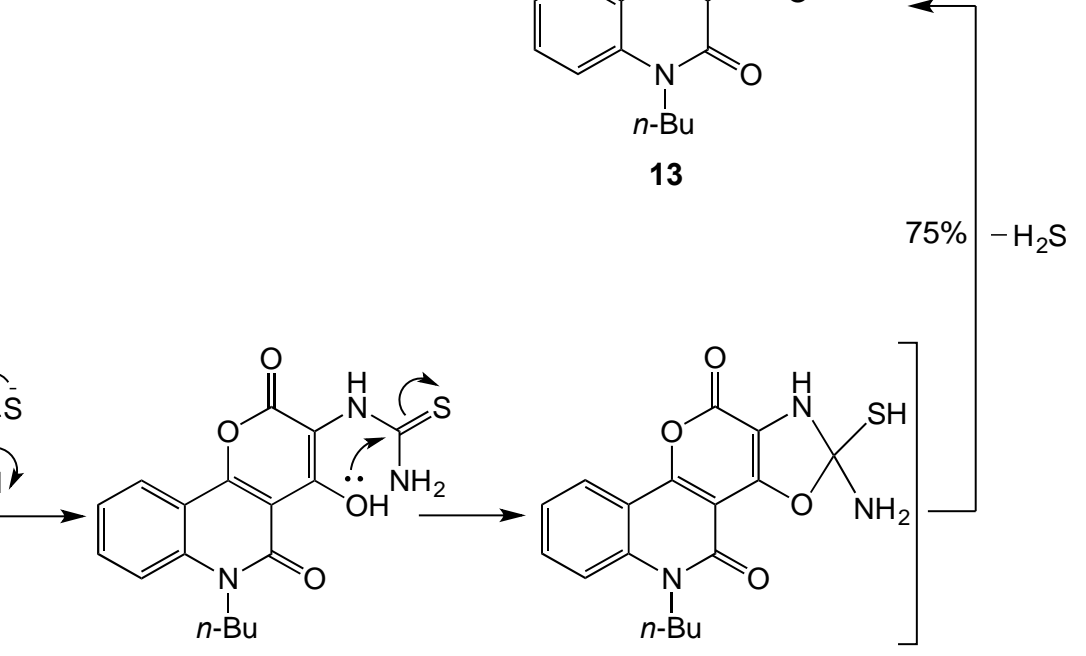

\section{Scheme 5}

The ${ }^{1} \mathrm{H}-\mathrm{NMR}$ spectrum of product 13 showed the presence of two deuterium-exchangeable protons, as a broad singlet signal at $7.71 \mathrm{ppm}$ due to $\mathrm{NH}_{2}$ protons. The ${ }^{13} \mathrm{C}-\mathrm{NMR}$ spectrum of compound 13 revealed the presence of four $s p^{3}$ hybridized carbon atoms due to the butyl group and thirteen $s p^{2}$ hybridized carbon atoms belonging to the aromatic carbon atoms of oxazolopyrano quinolinone system. The mass spectrum of compound 13 gave a molecular ion peak at $m / z 325\left(\mathrm{M}^{+}\right)$corresponding to the molecular formula $\mathrm{C}_{17} \mathrm{H}_{15} \mathrm{~N}_{3} \mathrm{O}_{4}$.

A series of a novel fluoro derivatives of pyranoquinolinones $14 a-c$ were synthesized from the reaction of aminopyranoquinolinone $\mathbf{3}$ with some fluorobenzoyl chlorides in refluxing dry THF (Scheme 6).

Evidence for the formation of compounds 14a-c came from their IR spectra where there was characteristic absorption at ca. $3447-3389 \mathrm{~cm}^{-1}$ due to an $\mathrm{N}-\mathrm{H}$ bond, and a new stretching signal due to the $\mathrm{C}=\mathrm{O}$ group of the amide between 1639 to $1677 \mathrm{~cm}^{-1}$. The ESI-MS spectra of compounds 14b-c exhibited 
$[\mathrm{M}+\mathrm{Na}]^{+}$ions $(\mathrm{m} / \mathrm{z}: 445.0197,445.1098$ and 511.2984$)$ in accordance with their structures. Furthermore, the amide $(\mathrm{N}-\mathrm{H})$ proton of this series was observed between $9.53 \mathrm{ppm}$ to $9.81 \mathrm{ppm}$ as a singlet signal in the ${ }^{1} \mathrm{H}$ NMR spectra of compounds 14a-c. The aryl carbon atoms were observed between 100 and 162 ppm in their ${ }^{13}$ C NMR spectra.

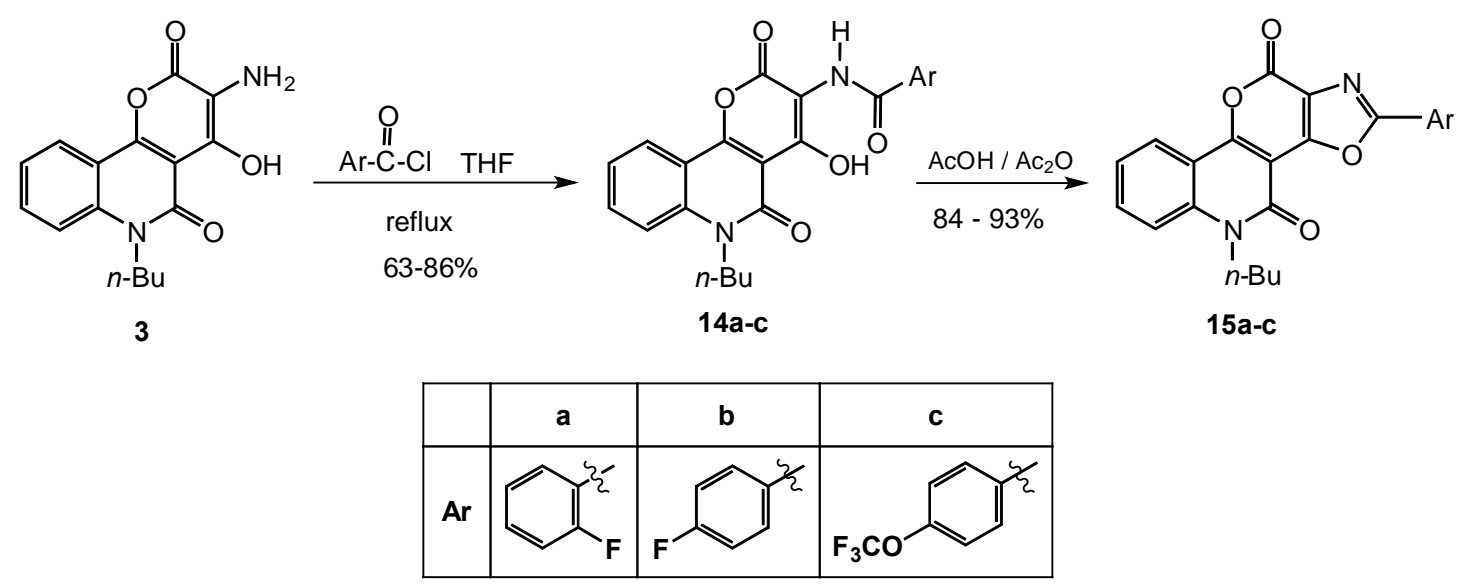

\section{Scheme 6}

Intramolecular heterocyclization of compounds 14 a-c by heating in a mixture of glacial acetic acid and acetic anhydride (80:20) gave our target fluoro derivatives 15a-c in good yields (Scheme 6). The IR spectra of all derivatives 15a-c showed the absence of the stretching absorption bands attributed to $\mathrm{OH}$ and $\mathrm{C}=\mathrm{O}$ amide. The ESI-MS spectrum of compound 15a shows a $[\mathrm{M}+\mathrm{H}]^{+}$ion at $\mathrm{m} / \mathrm{z} 405.1330$ and a $[\mathrm{M}+2 \mathrm{H}]^{2+}$ ion at 406.1340, the $[2 \mathrm{M}+\mathrm{Na}]^{+}$ion was also observed at $\mathrm{m} / \mathrm{z}$ 831.3452. ESI-MS spectrum of compound $15 \mathbf{b}$ showed an $[\mathrm{M}+\mathrm{H}]^{+}$ ion at $\mathrm{m} / \mathrm{z} 405.2520$ and the $[2 \mathrm{M}+\mathrm{Na}]^{+}$ion at $\mathrm{m} / \mathrm{z}$ 831.3457. The ESI-MS spectrum of compound $15 \mathrm{c}$ revealed a quasimolecular ion peak at $\mathrm{m} / \mathrm{z} 471.1[\mathrm{M}+\mathrm{H}]^{+}$and a sodiated molecular ion peak at $\mathrm{m} / \mathrm{z} 493.2[\mathrm{M}+\mathrm{Na}]^{+}$.

\section{Experimental Section}

General. TLC analysis of the reaction mixtures was performed using Fluka analytical silica gel $60 \mathrm{~F} 254 \mathrm{~nm}$ TLC plates. For column chromatography, Fluka analytical silica gel 60 0.063-0.2 mm (70-230 mesh ASTM) was used for separations. Melting points were recorded on Sanyo Gallenkamp MPD 350-BM 3.5 Melting Point apparatus. A Thermo Nicolet Nexus 470 FT-IR spectrophotometer was used for IR analyses. ${ }^{1} \mathrm{H}-\mathrm{NMR}(400 \mathrm{MHz})$ and ${ }^{13} \mathrm{C}-\mathrm{NMR}(101 \mathrm{MHz})$ measurements were performed using Varian-400 $\mathrm{MHz}$ spectrometer, and chemical shifts are expressed in $\delta(\mathrm{ppm})$ relative to TMS (in $\mathrm{CDCl}_{3}$ or DMSO- $d_{6}$ as solvent) as the internal standard. Elemental microanalyses were performed Perkin-Elmer CHN-2400II at the Chemical War Department, Ministry of Defence, Cairo, Egypt. Mass spectra were recorded on a Gas Chromatographic GCMSqp 1000 ex Shimadzu instrument at $70 \mathrm{ev}$. For mass spectra, a triple-quadruple tandem mass spectrometer (Micromass W Quattro micro $^{T M}$, Waters Corp., Milford, MA, USA) or Waters ZMD Quadrupole equipped with electrospray ionization (ESI) were used. 
6-n-Butyl-3H-pyrano[3,2-c]quinoline-2,4,5(6H)-trione (1). Yield (92\%), mp 227-229 ${ }^{\circ} \mathrm{C},\left(\mathrm{mp} 228-230{ }^{\circ} \mathrm{C}\right) .{ }^{24}$

6-n-Butyl-4-hydroxy-3-nitro-2H-pyrano[3,2-c]quinoline-2,5(6H)-dione (2). To a solution of compound 1 (2.85 $\mathrm{g}, 10 \mathrm{mmol})$ in glacial $\mathrm{AcOH}$ acid $(50 \mathrm{~mL})$, kept under $50{ }^{\circ} \mathrm{C}$, a mixture of conc. $\mathrm{HNO}_{3}(2.5 \mathrm{~mL})$ and conc. $\mathrm{H}_{2} \mathrm{SO}_{4}$ $(2.5 \mathrm{~mL})$ was added dropwise with continuous stirring. The precipitate formed was filtered off, dried and crystallized from glacial AcOH to furnish 2 as pale brown needles $(2.7 \mathrm{~g}, 82 \%), \mathrm{mp} 203-205^{\circ} \mathrm{C}$. IR $\left(\mathrm{KBr}, \mathrm{cm}^{-1}\right)$ : 3444 broad band (OH), 3087 ( $\left.\mathrm{CH}_{\text {aromatic }}\right), 2961,2932,2872\left(\mathrm{CH}_{\text {aliphatic. }}\right), 1742\left(\mathrm{C}=\mathrm{O}_{\alpha \text {-pyrone }}\right), 1672\left(\mathrm{C}=\mathrm{O}_{\text {quinolone }}\right)$ and $1566(\mathrm{C}=\mathrm{C}) .{ }^{1} \mathrm{H}$ NMR $\left(400 \mathrm{MHz}, \mathrm{CDCl}_{3}\right) \delta_{H}: 1.01(\mathrm{t}, \mathrm{J} 8.0 \mathrm{~Hz}, 3 \mathrm{H}, \mathrm{C} 4), 1.45-1.54\left(\mathrm{~m}, 2 \mathrm{H}, \mathrm{C}^{\prime}\right), 1.71-1.79(\mathrm{~m}, 2 \mathrm{H}$, $\left.\mathrm{C}^{\prime}\right), 4.33\left(\mathrm{t}, 2 \mathrm{H}, \mathrm{J} 8.0 \mathrm{~Hz}, \mathrm{C} 1^{\prime}\right), 7.39(\mathrm{t}, 1 \mathrm{H}, J 8.0 \mathrm{~Hz}, \mathrm{C} 9-\mathrm{H}), 7.46(\mathrm{~d}, J 8.0 \mathrm{~Hz}, 1 \mathrm{H}, \mathrm{C} 7-\mathrm{H}), 7.65(\mathrm{t}, 1 \mathrm{H}, J 8.0 \mathrm{~Hz}, \mathrm{C} 8-$ $\mathrm{H}), 8.24(\mathrm{dd}, 1 \mathrm{H}, J$ 8.0, $4.0 \mathrm{~Hz}, \mathrm{C} 10-\mathrm{H}), 12.38(\mathrm{~s}, 1 \mathrm{H}, \mathrm{OH}) .{ }^{13} \mathrm{C} \mathrm{NMR}\left(101 \mathrm{MHz}, \mathrm{CDCl}_{3}\right) \delta_{\mathrm{C}}: 13.7$ (s, C4 ), $20.1(\mathrm{~s}$, C3'), 29.5 (s, C2 '), 42.8 (s, C1 '), 97.8 (s, C3), 113.1 (s, C4a), 115.7 (s, C10a), 118.4 (s, C7), 125.1 (s, C10), 125.5 (s, C8), 136.0 (s, C6a), 138.9 (s, C9), 153.4 (s, C10b), 159.3 (s, C4), 163.1 (s, C2), 165.4 (s, C5). ESI-MS m/z: $331.1835[\mathrm{M}+\mathrm{H}]^{+}, 353.1434[\mathrm{M}+\mathrm{Na}]^{+}, 354.2070[\mathrm{M}+\mathrm{Na}+\mathrm{H}]^{2+}, 386.7095[2 \mathrm{M}+\mathrm{Na}]^{+}$. Anal. Calcd for $\mathrm{C}_{16} \mathrm{H}_{14} \mathrm{~N}_{2} \mathrm{O}_{6}$ (330.30): C, 58.18; H, 4.27; N, 8.48. Found C, 57.52; H, 4.20; N, 8.39\%.

3-Amino-6-n-butyl-4-hydroxy-2H-pyrano[3,2-c]quinoline-2,5(6H)-dione (3). A mixture of compound 2, (10 $\mathrm{mmol})$ tin metal powder $(4.00 \mathrm{~g}, 33 \mathrm{mmol})$ and conc. $\mathrm{HCl}(30 \mathrm{~mL})$ was stirred at $130{ }^{\circ} \mathrm{C}$ for $1 \mathrm{~h}$. MeOH $(25 \mathrm{~mL})$ was added to the reaction mixture and the mixture heated under reflux for $2 \mathrm{~h}$ until the color became clear yellow. The reaction mixture was filtered hot and the filtrate poured onto ice $(200 \mathrm{~g})$. The obtained solid was filtered off, dried under vacuum and crystallized from EtOH (96\%) to produce 3 as yellow crystals (2.4 g, 79\%),

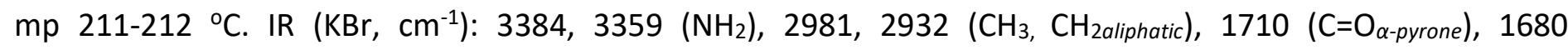
$\left(\mathrm{C}=\mathrm{O}_{\text {quinolone }}\right)$ and 1608 ( $\left.\mathrm{C}=\mathrm{C}_{\text {aliphatic }}\right) .{ }^{1} \mathrm{H} \mathrm{NMR}\left(400 \mathrm{MHz}, \mathrm{CDCl}_{3}\right) \delta_{H}: 0.98\left(\mathrm{t}, J 8.0 \mathrm{~Hz}, 3 \mathrm{H}, \mathrm{C} 4^{\prime}\right), 1.42-1.49(\mathrm{~m}, 2 \mathrm{H}$, $\left.\mathrm{C}^{\prime}\right), 1.71-1.75\left(\mathrm{~m}, 2 \mathrm{H}, \mathrm{C} 2{ }^{\prime}\right), 3.72\left(\mathrm{bs}, 2 \mathrm{H}, \mathrm{NH}_{2}\right), 4.28\left(\mathrm{t}, 2 \mathrm{H}, J 8 \mathrm{~Hz}, 1^{`} \mathrm{C}\right), 7.33(\mathrm{t}, 1 \mathrm{H}, \mathrm{J} 8.0 \mathrm{~Hz}, \mathrm{C} 9-\mathrm{H}), 7.38(\mathrm{~d}, 1 \mathrm{H}$, J $8.0 \mathrm{~Hz}, \mathrm{C} 7-\mathrm{H}), 7.61(\mathrm{t}, 1 \mathrm{H}, J 8.0 \mathrm{~Hz}, \mathrm{C} 8-\mathrm{H}), 8.12(\mathrm{dd}, 1 \mathrm{H}, J 1.2,8.00 \mathrm{~Hz}, \mathrm{C} 10-\mathrm{H}), 12.31(\mathrm{~s}, 1 \mathrm{H}, \mathrm{OH}$ exchangeable

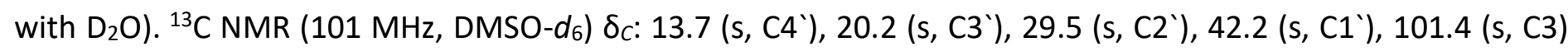
113.9 (s, C4a), 114.8 (s, C7), 114.2 (s, C10a), 123.4 (s, C10), 123.7 (s, C9), 131.7 (s, C8), 136.2 (s, C6a), 146.1 (s, C10b), 159.1 (s, C4), 149.8 (s, C2), 162.1 (s, C5). m/z (/r \%): $302\left(\mathrm{M}^{+}+2,2\right), 301\left(\mathrm{M}^{+}+1,19\right), 300$ (M+, 100), 299 $\left(\mathrm{M}^{+}-1,2\right), 244(40), 161(75)$. ESI-MS m/z: $301.2[\mathrm{M}+\mathrm{H}]^{+}, 323.1[\mathrm{M}+\mathrm{Na}]^{+}$. Anal. Calcd for $\mathrm{C}_{16} \mathrm{H}_{16} \mathrm{~N}_{2} \mathrm{O}_{4}(300.32): \mathrm{C}$, 63.99; H, 5.37; N, 9.33. Found C, 63.72; H, 5.26; N, 9.29\%.

\section{5-n-Butyl-4H-oxazolo[5',4':4,5]pyrano[3,2-c]quinoline-4,11(5H)-dione (5).}

Method a. A mixture of compound $3(3 \mathrm{~g}, 10 \mathrm{mmol})$ and triethyl orthoformate $(8 \mathrm{~mL}, 50 \mathrm{mmol})$ was heated for $12 \mathrm{~h}$. The progress of the reaction was monitored by TLC. The solid deposited after cooling was filtered off then washed with hot EtOH then crystallized from glacial $\mathrm{AcOH}$ to afford compound $\mathbf{5}$ (1.31 g, 42\%).

Method b: A mixture of compound 3 ( $3 \mathrm{~g}, 10 \mathrm{mmol}$ ) and formic acid $(25 \mathrm{~mL})$, was heated for $6 \mathrm{~h}$. The deposited precipitate was filtered off, air dried and crystallized from AcOH to give compound 5 (1.68 g, $54 \%)$.

Method c. A mixture of compound $6(3.55 \mathrm{~g}, 10 \mathrm{mmol})$ and glacial acetic acid was heated under reflux for $6 \mathrm{~h}$. Progress of the reaction was monitored by TLC. The reaction mixture was filtered and the filtrate poured onto ice. The obtained solid was filtered off, washed with water $(3 \times 20 \mathrm{~mL})$, then $\mathrm{Et}_{2} \mathrm{O}(3 \times 20 \mathrm{~mL})$ and crystallized from $\mathrm{AcOH}$ to give compound 5 as yellow crystals $(2.49 \mathrm{~g}, 80 \%), \mathrm{mp} 237-239{ }^{\circ} \mathrm{C}$. IR $\left(\mathrm{KBr}, \mathrm{cm}^{-1}\right): 3451$ broad band $(\mathrm{OH}), 3078$ ( $\left.\mathrm{CH}_{\text {aromatic }}\right), 2955,2917,2848$ ( $\left.\mathrm{CH}_{\text {aliphatic. }}\right), 1742\left(\mathrm{C}=\mathrm{O}_{\alpha \text {-pyrone }}\right), 1669$ ( $\left.\mathrm{C}=\mathrm{O}_{\text {quinolone }}\right)$ and 1646 $\left(\mathrm{C}=\mathrm{N}_{\text {oxazolo }}\right), 1591\left(\mathrm{C}=\mathrm{C}_{\text {aromatic }}\right) .{ }^{1} \mathrm{H}$ NMR (DMSO- $\left.d_{6}, \delta, 400 \mathrm{MHz}\right) \delta_{H}: 0.90(\mathrm{t}, J 8.0 \mathrm{~Hz}, 3 \mathrm{H}, \mathrm{C} 4), 1.34-1.44(\mathrm{~m}, 2 \mathrm{H}$, $\left.\mathrm{C}^{\prime}\right), 1.59-1.68\left(\mathrm{~m}, 2 \mathrm{H}, \mathrm{C} 2^{\prime}\right), 4.36\left(\mathrm{t}, 2 \mathrm{H}, J 8 \mathrm{~Hz}, 1^{`} \mathrm{C}\right), 7.57(\mathrm{t}, 1 \mathrm{H}, J 8.0 \mathrm{~Hz}, \mathrm{C} 9-\mathrm{H}), 7.81(\mathrm{~d}, 1 \mathrm{H}, J 8.0 \mathrm{~Hz}, \mathrm{C} 7-\mathrm{H})$, $7.91(\mathrm{t}, 1 \mathrm{H}, J 8.0 \mathrm{~Hz}, \mathrm{C} 8-\mathrm{H}), 8.20(\mathrm{dd}, 1 \mathrm{H}, J 1.2,8.00 \mathrm{~Hz}, \mathrm{C} 10-\mathrm{H}), 8.79\left(\mathrm{~s}, 1 \mathrm{H}, \mathrm{CH}_{\text {oxazole }}\right) .{ }^{13} \mathrm{C} \mathrm{NMR}(101 \mathrm{MHz}, \mathrm{DMSO}-$ $\left.d_{6}\right) \delta_{c}: 14.1\left(\mathrm{~s}, \mathrm{C}^{\prime}\right), 19.8\left(\mathrm{~s}, \mathrm{C} 3^{\prime}\right), 29.6\left(\mathrm{~s}, \mathrm{C} 2^{\prime}\right), 42.4$ (s, C1 $\left.{ }^{\prime}\right), 100.7$ (s, C4a) 113.7 (s, C7), 116.6 (s, C10a), 124.4 (s, C10), 133.5 (s, C9), 135.5 (s, 1C, 3), 137.0 (s, 1C, 8), 138.2 (s, C6a), 144.1 (s, C4), 145.8 (s, C2 oxazolo), 158.7 (s, 
C10b), 162.8 (s, C,2), 163.7 (s, C5). m/z (/r\%): 311 ( $\left.\mathrm{M}^{+}+1,10\right), 310$ (M+, 30), 243 (42), 188 (95), 132 (100). Anal. Calcd for $\mathrm{C}_{17} \mathrm{H}_{14} \mathrm{~N}_{2} \mathrm{O}_{4}$ (310.31): C, 65.80; $\mathrm{H}, 4.55 ; \mathrm{N}, 9.03$. Found $\mathrm{C}, 65.52 ; \mathrm{H}, 4.20 ; \mathrm{N}, 8.99 \%$.

\section{$N^{\prime}$-(6-n-Butyl-4-hydroxy-2,5-dioxo-5,6-dihydro-2H-pyrano[3,2-c]quinolin-3-yl)- $\mathbf{N}, \mathbf{N}$-dimethylformimidamide}

(6). A mixture of compound $3(3 \mathrm{~g}, 10 \mathrm{mmol})$ and DMF-DMA $(3.0 \mathrm{~mL}, 10 \mathrm{mmol})$ in $(50 \mathrm{~mL})$ dry toluene was heated under reflux for $6 \mathrm{~h}$. The progress of the reaction was monitored by TLC. After completion of reaction, the solvent was removed under vacuum to produce a yellow oily residue. The targeted compound 4 was obtained using column chromatography (ethyl acetate/hexane 6:4) as yellow crystals (2.98 g, 84\%), mp 192$193{ }^{\circ} \mathrm{C}$. IR $\left(\mathrm{KBr}, \mathrm{cm}^{-1}\right)$ : 3409 broad band $(\mathrm{OH}), 2953,2918,2866$ (CH aliphatic.), 1715(C=O $\alpha$-pyrone), 1650 $\left(\mathrm{C}=\mathrm{O}_{\text {quinolone }}\right)$ and $1613(\mathrm{C}=\mathrm{N}$ imid $), 1539$ ( $\left.\mathrm{C}=\mathrm{C}_{\text {aromatic }}\right) .{ }^{1} \mathrm{H}$ NMR (DMSO- $\left.d_{6}\right) \delta_{H}: 0.91(\mathrm{t}, J$ 8Hz, 3H, C4 $), 1.33-1.42(\mathrm{~m}$, $\left.2 \mathrm{H}, \mathrm{C} 3^{\prime}\right), 1.54-1.61\left(\mathrm{~m}, 2 \mathrm{H}, \mathrm{C} 2{ }^{\prime}\right), 3.05\left(\mathrm{~s}, 3 \mathrm{H}, \mathrm{N}-\mathrm{CH}_{3}\right), 3.14\left(\mathrm{~s}, 3 \mathrm{H}, \mathrm{N}-\mathrm{CH}_{3}\right), 4.21\left(\mathrm{t}, 2 \mathrm{H}, J 8 \mathrm{~Hz}, \mathrm{C} 1^{\prime}\right), 7.34(\mathrm{t}, 1 \mathrm{H}, J$ $8 \mathrm{~Hz}, \mathrm{C} 9-\mathrm{H}), 7.60(\mathrm{~d}, 1 \mathrm{H}, J 8 \mathrm{~Hz}, \mathrm{C} 7-\mathrm{H}), 7.70(\mathrm{t}, J 8 \mathrm{~Hz}, 1 \mathrm{H}, \mathrm{C} 8-\mathrm{H}), 8.04(\mathrm{dd}, 1 \mathrm{H}, J 1.2,8 \mathrm{~Hz}, \mathrm{C} 10-\mathrm{H}), 8.16(\mathrm{~s}, 1 \mathrm{H}$,

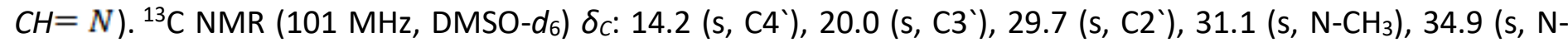
$\mathrm{CH}_{3}$ ), 35.6 (s, C1'), 105.9 (s, C3), 113.8 (s, C4a), 115.5 (s, C10a), 122.9 (s, C7), 123.9 (s, C10), 133.0 (s, C9), 138.4 (s, C8), 156.3, (s, C6a), 157.1 (s, C10b), 159.5 (s, C2), 159.9 (s, C4), 160.5 (s, C5), 207.0 (s, N=CH). ESI-MS m/z: $356.2[\mathrm{M}+\mathrm{H}]^{+}, 357.2[\mathrm{M}+2 \mathrm{H}]^{2+}, 379.1[\mathrm{M}+\mathrm{H}+\mathrm{Na}]^{2+}$. Anal. Calcd for $\mathrm{C}_{19} \mathrm{H}_{21} \mathrm{~N}_{3} \mathrm{O}_{4}$ (355.40): C, 64.21; $\mathrm{H}, 5.96 ; \mathrm{N}$, 11.82. Found C, 64.19; $H, 5.20 ; N, 11.79 \%$.

\section{5-n-Butyl-2-methyl-4H-oxazolo[5',4':4,5]pyrano[3,2-c]quinoline-4,11(5H)-dione (8).}

Method a. A mixture of compound 3 ( $3 \mathrm{~g}, 10 \mathrm{mmol})$ and triethyl orthoacetate $(9 \mathrm{~mL}, 50 \mathrm{mmol})$ was heated for $12 \mathrm{~h}$. The progress of the reaction was monitored by TLC. The solid deposited after cooling was filtered off and washed with hot EtOH. The targeted compound crystallized from $\mathrm{AcOH}$ to give compound 8 as pale brown crystals (1.45 g, 46\%).

Method b. A mixture of compound 3 ( $3 \mathrm{~g}, 10 \mathrm{mmol}$ ) and $\mathrm{Ac}_{2} \mathrm{O}(25 \mathrm{~mL})$, was heated for $6 \mathrm{~h}$. The solid deposited was filtered off, air dried and crystallized from $\mathrm{AcOH}$ to give compound 8 (1.64 g, 52\%).

Method c. To a mixture of 9 (3.4 g, $10 \mathrm{mmol})$ in glacial AcOH (80\%) $(40 \mathrm{~mL}), \mathrm{Ac}_{2} \mathrm{O}(20 \%)(10 \mathrm{~mL}) \mathrm{was}$ added dropwise with stirring at $\mathrm{rt}$ for a period of $(10 \mathrm{~mL})$ then heated under reflux till the reaction was complete as judged by TLC $(24 \mathrm{~h})$. The reaction mixture was cooled to rt and poured on ice ( $200 \mathrm{~g})$. The brown precipitate was filtered off, washed with water $(3 \times 20 \mathrm{~mL})$, dried and crystallized from $\mathrm{AcOH}$ to give compound 8 as

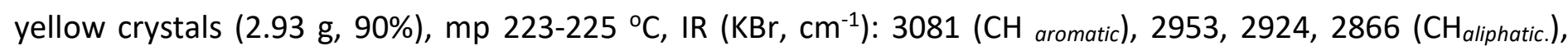
1755 ( $\left.\mathrm{C}=\mathrm{O}_{\alpha \text {-pyrone }}\right), 1650$ ( $\mathrm{C}=\mathrm{O}$ quinolone, $)$ and 1623 ( $\left.\mathrm{C}=\mathrm{N}_{\text {oxazolo }}\right), 1586\left(\mathrm{C}=\mathrm{C}_{\text {aromatic }}\right) .{ }^{1} \mathrm{H} \mathrm{NMR}\left(400 \mathrm{MHz} \mathrm{CDCl}_{3}\right) \delta_{H}$ : $1.00\left(\mathrm{t}, 3 \mathrm{H}, \mathrm{J} 8.00 \mathrm{~Hz}, \mathrm{C} 4^{\prime}\right), 1.46-1.57\left(\mathrm{~m}, 2 \mathrm{H}, \mathrm{C} 3{ }^{\prime}\right), 1.71-1.81\left(\mathrm{~m}, 2 \mathrm{H}, \mathrm{C} 2{ }^{\prime}\right), 2.73\left(\mathrm{~s}, 3 \mathrm{H}, \mathrm{CH}_{3}\right.$ oxazolo), $4.36(\mathrm{t}, 2 \mathrm{H}$, J $\left.8.00 \mathrm{~Hz}, \mathrm{C} 1^{\prime}\right), 7.37(\mathrm{t}, 1 \mathrm{H}, J 8.00 \mathrm{~Hz}, \mathrm{C} 9-\mathrm{H}), 7.45(\mathrm{~d}, 1 \mathrm{H}, J 8.00 \mathrm{~Hz}, \mathrm{C} 7-\mathrm{H}), 7.71(\mathrm{t}, 1 \mathrm{H}, J 8.00 \mathrm{~Hz}, \mathrm{C} 8-\mathrm{H}), 8.32$ (dd, $1 \mathrm{H}, J$ 8.00, $1.17 \mathrm{~Hz}, \mathrm{C} 10-\mathrm{H}) .{ }^{13} \mathrm{C} N M R\left(101 \mathrm{MHz}, \mathrm{DMSO}-d_{6}\right) \delta_{c}: 13.8\left(\mathrm{~s}, \mathrm{CH}_{3 \text { oxazolo }}\right), 14.4\left(\mathrm{~s}, \mathrm{C} 4^{\prime}\right), 20.2$ (s, C3'), 29.6 (s, C2 ) , 42.5 (s, C1 '), 100.8 (s, C3), 113.5 (s, C10a), 114.9 (s, C7), 123.2 (s, C10), 124.7 (s, C9), 124.8 (s, C4a), 133.4 (s, C8), 138.7 (s, C6a), 154.5 (s, C4), 155.9 (s, C2 oxazolo), 156.7 (s, C10b), 156.9 (s, C2), 164.7(s, C5). ESI-MS m/z: $325.3[\mathrm{M}+\mathrm{H}]^{+}, 347.1[\mathrm{M}+\mathrm{Na}]^{+}, 671.1[2 \mathrm{M}+\mathrm{Na}]^{+}$. Anal. Calcd for $\mathrm{C}_{18} \mathrm{H}_{16} \mathrm{~N}_{2} \mathrm{O}_{4}(324.34): \mathrm{C}, 66.66 ; \mathrm{H}$, 4.97; N, 8.64. Found C, 66.19; $H, 4.99 ; N, 8.59 \%$.

$\mathbf{N}$-(6-n-Butyl-4-hydroxy-2,5-dioxo-5,6-dihydro-2H-pyrano[3,2-c]quinolin-3-yl)acetamide (9). To a solution of compound 3 ( $3 \mathrm{~g}, 10 \mathrm{mmol})$ in THF $(50 \mathrm{~mL})$, acetyl chloride $(0.75 \mathrm{~mL}, 10 \mathrm{mmol})$ was added and the mixture was heated under reflux for $4 \mathrm{~h}$. After completion of reaction (as indicated by the disappearance of starting material on TLC) the reaction mixture was poured onto crushed ice (100 g). The separated solid was filteredoff, washed with water $(3 \times 10 \mathrm{~mL})$, dried and crystallized from EtOH to give compound 9 as off white crystals (3.25 g, 95\%), mp 185-187 ${ }^{\circ} \mathrm{C}$. IR ( $\left.\mathrm{KBr}, \mathrm{cm}^{-1}\right): 3415$ (broad band, OH), $3082(\mathrm{NH}), 3050\left(\mathrm{CH}_{\text {aromatic }}\right), 2953$, 2924, 2866 ( $\left.\mathrm{CH}_{\text {aliphatic. }}\right), 1738$ ( $\left.\mathrm{C}=\mathrm{O}_{\alpha \text {-pyrone }}\right), 1703$ ( $\left.\mathrm{C}=\mathrm{O}_{\text {acetyll }}\right), 1668$ ( $\left.\mathrm{C}=\mathrm{O}_{\text {quinolone, }}\right)$ and $1568\left(\mathrm{C}=\mathrm{C}_{\text {aromatic }}\right) .{ }^{1} \mathrm{H} \mathrm{NMR}$ $\left(400 \mathrm{MHz}, \mathrm{DMSO}-d_{6}\right) \delta_{H}: 0.91\left(\mathrm{t}, 3 \mathrm{H}, \mathrm{J} 8.00 \mathrm{~Hz}, \mathrm{C} 4{ }^{\prime}\right), 1.34-1.46\left(\mathrm{~m}, 2 \mathrm{H}, \mathrm{C} 3^{\prime}\right), 1.59-1.70\left(\mathrm{~m}, 2 \mathrm{H}, \mathrm{C} 2^{\prime}\right), 2.06(\mathrm{~s}, 3$ 
$\left.\mathrm{H}, \mathrm{CH}_{3 \text { acetyl }}\right), 4.34\left(\mathrm{t}, 2 \mathrm{H}, \mathrm{J} 8.00 \mathrm{~Hz}, \mathrm{C} 1^{\prime}\right), 7.52(\mathrm{t}, 1 \mathrm{H}, \mathrm{J} 8.00 \mathrm{~Hz}, \mathrm{C} 9-\mathrm{H}), 7.73(\mathrm{~d}, 1 \mathrm{H}, \mathrm{J} 8.00 \mathrm{~Hz}, \mathrm{C} 7-\mathrm{H}), 7.85$ (t, $1 \mathrm{H}, J$ $8.00 \mathrm{~Hz}, \mathrm{C} 8-\mathrm{H}), 8.14(\mathrm{dd}, 1 \mathrm{H}, \mathrm{J} 8.00,1.17 \mathrm{~Hz}, \mathrm{C} 10-\mathrm{H}), 9.12\left(\mathrm{~s}, 1 \mathrm{H}, \mathrm{N}-\mathrm{H}_{\text {amide }}\right.$ exchangeable in $\left.\mathrm{D}_{2} \mathrm{O}\right), 13.64(\mathrm{bs}, 1 \mathrm{H}$, C4-OH exchangeable in $\left.D_{2} O\right) .{ }^{13} \mathrm{C} \mathrm{NMR}\left(101 \mathrm{MHz} \mathrm{CDCl}_{3}\right) \delta_{\mathrm{c}}: 13.7$ (s, C4 ), 20.1 (s, C3'), $23.2\left(\mathrm{~s}, \mathrm{CH}_{3 \text { acetyl) }}, 29.5\right.$ (s, C2'), 42.4 (s, C1'), 100.0 (s, C3), 101.6 (s, C4a), 113.6 (s, C10a), 115.1 (s, C7), 124.2 (s, C10) 124.8 (s, C9), 134.0 (s, C8), 137.9 (s, C6a), 156.9 (s, C4), 159.7 (s, C10b), 162.8 (s, C2), 169.0 (s, C5), 207.0 (s, C=O acetyle). ESI-MS m/z: $343.1[\mathrm{M}+\mathrm{H}]^{+}, 365.1[\mathrm{M}+\mathrm{Na}]^{+}$. Anal. Calcd for $\mathrm{C}_{18} \mathrm{H}_{18} \mathrm{~N}_{2} \mathrm{O}_{5}$ (342.35): C, 63.15; $\mathrm{H}, 5.30 ; \mathrm{N}, 8.18$. Found C, 62.81; $\mathrm{H}$, $5.20 ; \mathrm{N}, 8.16 \%$.

1-(6-n-Butyl-4-hydroxy-2,5-dioxo-5,6-dihydro-2H-pyrano[3,2-c]quinolin-3-yl)-3-phenylthiourea (10). To a solution of compound 3 ( $3 \mathrm{~g}, 10 \mathrm{mmol})$ and pyridine $(1 \mathrm{~mL})$ in absolute EtOH $(50 \mathrm{~mL})$, phenyl isothiocyanate $(1.21 \mathrm{~mL}, 10 \mathrm{mmol})$ was added. The reaction mixture was heated under reflux for $6 \mathrm{~h}$. The reaction was monitored by TLC. The reaction mixture was filtered hot and the filtrate was poured on ice (200 g). The obtained solid was filtered off, washed with water $(3 \times 10 \mathrm{~mL})$, dried and crystallized from DMF to give compound 10 as a pale yellow powder $(2.8 \mathrm{~g}, 65 \%), \mathrm{mp} 126-129{ }^{\circ} \mathrm{C}$. IR $\left(\mathrm{KBr}, \mathrm{cm}^{-1}\right): 3442$ broad band $(\mathrm{OH})$,

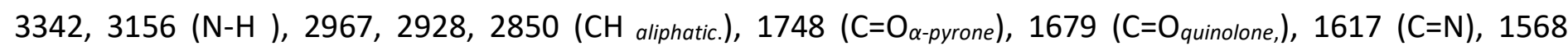
(C=C aromatic) and $1283(\mathrm{C}=\mathrm{S}) .{ }^{1} \mathrm{H}$ NMR $\left(400 \mathrm{MHz}, \mathrm{DMSO}-d_{6}\right) \delta_{H}: 0.90(\mathrm{t}, J 8.00 \mathrm{~Hz}, 3 \mathrm{H}, \mathrm{C} 4), 1.34-1.43(\mathrm{~m}, 2 \mathrm{H}$, $\left.\mathrm{C}^{\prime}\right), 1.56-1.66\left(\mathrm{~m}, 2 \mathrm{H}, \mathrm{C} 2^{\prime}\right), 4.33\left(\mathrm{t}, 2 \mathrm{H}, \mathrm{J} 8.00 \mathrm{~Hz}, \mathrm{C} 1^{\prime}\right), 7.05-7.14\left(\mathrm{~m}, 1 \mathrm{H}, \mathrm{C}-\mathrm{H}_{\text {phenyl }}\right), 7.29(\mathrm{t}, 1 \mathrm{H}, J 8.00 \mathrm{~Hz}$,

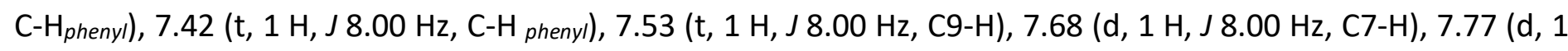
$\mathrm{H}, J 8.00 \mathrm{~Hz}, \mathrm{C}-\mathrm{H}_{\text {phenyl) }}, 7.85(\mathrm{t}, 1 \mathrm{H}, J 8.00 \mathrm{~Hz}, \mathrm{C} 8-\mathrm{H}), 8.01$ (d, $1 \mathrm{H}, J 8.00 \mathrm{~Hz}, \mathrm{C}-\mathrm{H}_{\text {phenyl) }}, 8.15$ (dd, $1 \mathrm{H}, J 8.00,1.17$ $\mathrm{Hz}, \mathrm{C} 10-\mathrm{H}), 8.94\left(\mathrm{~s}, 1 \mathrm{H}, \mathrm{N}-\mathrm{H}\right.$ exchangeable in $\left.\mathrm{D}_{2} \mathrm{O}\right), 9.76\left(\mathrm{~s}, 1 \mathrm{H}, \mathrm{N}-\mathrm{H}\right.$ exchangeable in $\left.D_{2} \mathrm{O}\right), 13.73(\mathrm{bs}, 1 \mathrm{H}, \mathrm{OH}$ exchangeable in $\left.D_{2} O\right) .{ }^{13} \mathrm{C} N M R\left(101 \mathrm{MHz}, \mathrm{DMSO}-d_{6}\right) \delta_{c}: 14.1\left(\mathrm{~s}, \mathrm{C} 4^{\prime}\right), 19.9\left(\mathrm{~s}, \mathrm{C} 3^{\prime}\right), 29.6\left(\mathrm{~s}, \mathrm{C} 2^{\prime}\right), 42.0\left(\mathrm{~s}, \mathrm{C} 1^{\prime}\right)$, 101.6 (s, C3), 113.9 (s, C4a), 114.5 (s, C7), 116.4 (s, C10a), 122.7 (s, C phenyl), 123.6 (s, C 10), 123.8 (s, C9), 124.1 (s, $\left.C_{\text {phenyl }}\right), 124.4$ (s, $\left.C_{\text {phenyl }}\right), 124.8$ (s, $C_{\text {phenyl) }}, 132.2$ (s, C8), 136.1 (s, C6a), 139.8 (s, $\left.C_{\text {phenyl }}\right), 149.0$ (s, C10b), 155.9 (s, C4), 159.3 (s, C2), 162.7 (s, C5), 163.0 (s, C=S). MS: m/z (relative intensity): 436 [M+ +1; 5], 435 [M+; 15], 189 (100). Anal. Calcd for $\mathrm{C}_{23} \mathrm{H}_{21} \mathrm{~N}_{3} \mathrm{O}_{4} \mathrm{~S}$ (435.51): C, 63.43; $\mathrm{H}, 4.86 ; \mathrm{N}, 9.65 ; \mathrm{S}, 7.36$. Found $\mathrm{C}, 63.32 ; \mathrm{H}, 4.76 ; \mathrm{N}, 9.36$; S, $7.23 \%$.

\section{5-n-Butyl-2-thioxo-4H-oxazolo[5',4':4,5]pyrano[3,2-c]quinoline-4,11(5H)-dione (12)}

Method a. To a solution of compound 10 ( $4.3 \mathrm{~g}, 10 \mathrm{mmol})$ in dry toluene $(50 \mathrm{~mL})$, $p$-toluenesulfonic acid $(0.3$ g) was added. The reaction mixture was heated under reflux for $24 \mathrm{~h}$. The progress of the reaction was monitored by TLC. The reaction mixture filtered and poured on ice (200 g). The obtained precipitate was filtered off, washed with water $(3 \times 10 \mathrm{~mL})$, dried and crystallized from EtOH to produce 12 as light brown crystals (1.95 g, 57\%), mp 163-165 ${ }^{\circ} \mathrm{C} . \mathrm{IR}\left(\mathrm{KBr}, \mathrm{cm}^{-1}\right): 3342,3100(\mathrm{~N}-\mathrm{H}), 3056\left(\mathrm{CH}_{\text {aromatic }}\right), 2928,2918,2849$

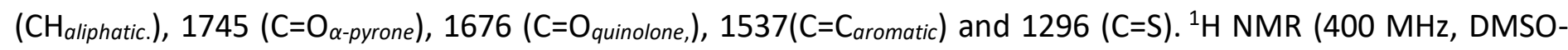
$\left.d_{6}\right) \delta_{H}: \operatorname{ppm} 0.92\left(\mathrm{t}, 3 \mathrm{H}, \mathrm{J} 8.00 \mathrm{~Hz}, \mathrm{C}^{\prime}\right), 1.33-1.45\left(\mathrm{~m}, 2 \mathrm{H}, \mathrm{C} 3^{\prime}\right), 1.55-1.65\left(\mathrm{~m}, 2 \mathrm{H}, \mathrm{C} 2^{\prime}\right), 4.26(\mathrm{t}, 2 \mathrm{H}, J 8.00 \mathrm{~Hz}$, C1 ) , 7.36 (t, $1 \mathrm{H}, J 8.00 \mathrm{~Hz}, \mathrm{C} 9-\mathrm{H}), 7.48(\mathrm{~d}, 1 \mathrm{H}, \mathrm{J} 8.00 \mathrm{~Hz}, \mathrm{C} 7-\mathrm{H}), 7.77(\mathrm{t}, 1 \mathrm{H}, J 8.00 \mathrm{~Hz}, \mathrm{C} 8-\mathrm{H}), 8.05$ (dd, $1 \mathrm{H}, J$ 8.00, $0.8 \mathrm{~Hz}, \mathrm{C} 10-\mathrm{H}), 10.78\left(\mathrm{~s}, 1 \mathrm{H}, \mathrm{N}-\mathrm{H}\right.$ exchangeable in $\left.\mathrm{D}_{2} \mathrm{O}\right) .{ }^{13} \mathrm{C}$ NMR $\left(101 \mathrm{MHz}, \mathrm{DMSO}-d_{6}\right) \delta_{c}: \mathrm{ppm} 13.8(\mathrm{~s}$, C4'), 20.2 (s, C3'), 29.4 (s, C2') 42.2 (s, C1'), 135.6 (s, C3), 129.7 (s, C4a), 114.6 (s, C7), 119.0 (s, C10a), 122.6 (s, C10), 124.6 (s, C9), 133.7 (s, C8), 136.4 (s, C6a), 156.9 (s, C10b), 141.2 (s, C4), 157.7 (s, C2 ), 161.8 (s, C5), 177.9 (s, C=S thioxo). MS: $m / z$ (relative intensity): $343\left[\mathrm{M}^{+}+1 ; 9\right], 342\left[\mathrm{M}^{+} ; 50\right], 286\left[\mathrm{M}^{+}-\mathrm{Bu} ; 24\right]$. Anal. Calcd for $\mathrm{C}_{17} \mathrm{H}_{14} \mathrm{~N}_{2} \mathrm{O}_{4} \mathrm{~S}$ (342.38): C, 59.64; H, 4.12; N, 8.18; S, 9.37. Found C, 59.12; H, 4.10; N, 8.08; S, 9.20\%.

Method b. To a solution of compound 3 ( $3 \mathrm{~g}, 10 \mathrm{mmol})$ in pyridine $(50 \mathrm{~mL})$, carbon disulfide $(0.9 \mathrm{~mL}, 15 \mathrm{mmol})$ was added dropwise. The mixture was heated under reflux for $12 \mathrm{~h}$, monitored by TLC. The reaction mixture was filtered hot and the filtrate was poured onto ice (100 g). The resulting precipitate was filtered off, washed with water $(3 \times 10 \mathrm{~mL})$, dried and crystallized from EtOH to produce 12 as light brown crystals $(2.05 \mathrm{~g}, 60 \%)$, $\mathrm{mp} 163-165^{\circ} \mathrm{C}$. 
2-Amino-5-n-butyl-4H-oxazolo[5',4':4,5]pyrano[3,2-c]quinoline-4,11(5H)-dione (13). Compound 3 (3 g, 10 $\mathrm{mmol})$ was mixed with pyridine $(50 \mathrm{~mL})$ and ammonium thiocyanate $(1.2 \mathrm{~g}, 15 \mathrm{mmol})$. The resulted mixture was heated under reflux till the reaction was complete as judged by TLC $(24 \mathrm{~h})$. The reaction mixture was cooled to $\mathrm{rt}$ and poured on ice (200 g). The obtained precipitate was filtered off, washed with water ( $3 \times 10$ $\mathrm{mL})$, dried and crystallized from EtOH to furnish 13 as yellow crystals $(2.45 \mathrm{~g}, 75 \%), \mathrm{mp} 258-260^{\circ} \mathrm{C} . \mathrm{IR}\left(\mathrm{KBr}, \mathrm{cm}^{-}\right.$ $\left.{ }^{1}\right)$ : 3488, 3394 douple peaks ( $\left.\mathrm{NH}_{\text {2aromatic }}\right), 3086$ ( $\left.\mathrm{CH}_{\text {aromatic }}\right), 2959,2930,2864$ ( $\left.\mathrm{CH}_{\text {aliphatic. }}\right), 1710$ (C=O $\left.\mathrm{O}_{\text {apyrone }}\right), 1682$ $\left(\mathrm{C}=\mathrm{O}_{\text {quinolone }}\right), 1634$ ( $\left.\mathrm{C}=\mathrm{N}_{\text {oxazolo }}\right), 1586$ ( $\left.\mathrm{C}=\mathrm{C}_{\text {aromatic }}\right) .{ }^{1} \mathrm{H} \mathrm{NMR}\left(400 \mathrm{MHz}, \mathrm{DMSO}-d_{6}\right) \delta_{\mathrm{H}}: 0.89\left(\mathrm{t}, 3 \mathrm{H}, J 8.00 \mathrm{~Hz}, \mathrm{C} 4{ }^{\prime}\right)$, $1.28-1.45\left(\mathrm{~m}, 2 \mathrm{H}, \mathrm{C} 3^{\prime}\right), 1.50-1.67\left(\mathrm{~m}, 2 \mathrm{H}, \mathrm{C} 2^{\prime}\right), 4.28\left(\mathrm{t}, 2 \mathrm{H}, J 8.00 \mathrm{~Hz}, \mathrm{C} 1^{\prime}\right), 7.42(\mathrm{t}, 1 \mathrm{H}, J 8.00 \mathrm{~Hz}, \mathrm{C} 9-\mathrm{H}), 7.56$ (d, $1 \mathrm{H}, J 8.00 \mathrm{~Hz}, \mathrm{C} 7-\mathrm{H}), 7.71$ (bs, $\left.2 \mathrm{H}, \mathrm{C}-\mathrm{NH}_{20 x a z o l o}\right), 8.01(\mathrm{t}, 1 \mathrm{H}, J 8.00 \mathrm{~Hz}, \mathrm{C} 8-\mathrm{H}), 8.06$ (dd, $1 \mathrm{H}, J 8.00,1.2 \mathrm{~Hz}$, C10-H). ${ }^{13} \mathrm{C}$ NMR (101 MHz, DMSO-d $\left.d_{6}\right) \delta_{c}: 14.1$ (s, C4 ), 19.9 (s, C3'), 29.6 (s, C2 '), 42.0 (s, C1'), 101.6 (s, C4a), 114.0 (s, C10a), 116.3 (s, C7), 122.7 (s, C10), 124.3 (s, C9), 124.3 (s, C3), 132.2 (s, C8), 136.1 (s, C6a), 158.9 (s, C4), 158.9 (s, C10b), 162.8 (s, C2 ), 164.9 (s, C2 oxazolo ), 165.6 (s, C5). MS: m/z (relative intensity): 326 [M+ +1; 5], $325\left[\mathrm{M}^{+} ; 10\right], 309\left[\mathrm{M}^{+}-\mathrm{NH}_{2}+\mathrm{H} ; 6\right], 269$ [M+-Bu+H; 20], 144 (100). Anal. Calcd for $\mathrm{C}_{17} \mathrm{H}_{15} \mathrm{~N}_{3} \mathrm{O}_{4}$ (325.33): C, 62.76; $\mathrm{H}, 4.65 ; \mathrm{N}, 12.92$. Found $\mathrm{C}, 62.57 ; \mathrm{H}, 4.63 ; \mathrm{N}, 12.81 \%$.

General procedure for preparation of compounds 14a-c. To a solution of compound 3 ( $3 \mathrm{~g}, 10 \mathrm{mmol}) \mathrm{in} \mathrm{THF}$ $(25 \mathrm{~mL})$, an equivalent amount of 2-fluorobenzoyl chloride $(1.79 \mathrm{~mL})$, 4-fluorobenzoyl chloride $(1.60 \mathrm{~mL})$ or 4 trifluoromethoxybenzoyl chloride $(1.60 \mathrm{~mL})$ was added and the reaction mixture was heated under reflux overnight. The targeted compounds 14a-c were precipitated. The obtained solid was filtered off, dried and crystallized from EtOH to produce the corresponding compound 14.

N-(6-n-Butyl-4-hydroxy-2,5-dioxo-5,6-dihydro-2H-pyrano[3,2c]quinolin-3-yl)-2-fluorobenzamide (14a). Pale yellow powder (2.9 g, 72\%), mp 215-217 ${ }^{\circ} \mathrm{C}$. IR ( KBr, cm $\left.{ }^{-1}\right): 3398(\mathrm{O}-\mathrm{H}), 3290(\mathrm{NH}), 3068\left(\mathrm{CH}_{\text {aromatic }}\right), 2954,2930$, 2864 ( $\left.\mathrm{CH}_{\text {aliphatic. }}\right), 1738$ ( $\left.\mathrm{C}=\mathrm{O}_{\text {a-pyrone }}\right), 1666$ ( $\left.\mathrm{C}=\mathrm{O}_{\text {quinolone }}\right), 1615$ ( $\left.\mathrm{C}=\mathrm{O}_{\text {arylcarbonyl }}\right)$ and $1537\left(\mathrm{C}=\mathrm{C}_{\text {aromatic }}\right) .{ }^{1} \mathrm{H} \mathrm{NMR}(400$ $\left.\mathrm{MHz}, \mathrm{DMSO}-d_{6}\right) \delta_{H}: 0.92\left(\mathrm{t}, J 8.00 \mathrm{~Hz}, 3 \mathrm{H}, \mathrm{C} 4^{\prime}\right), 1.35-1.47\left(\mathrm{~m}, 2 \mathrm{H}, \mathrm{C} 3^{\prime}\right), 1.60-1.72\left(\mathrm{~m}, 2 \mathrm{H}, \mathrm{C} 2^{\prime}\right), 4.33(\mathrm{t}, J 8.00$ $\left.\mathrm{Hz}, 2 \mathrm{H}, \mathrm{C} 1^{\prime}\right), 7.28-7.35\left(\mathrm{~m}, 2 \mathrm{H}, \mathrm{C} 9-\mathrm{H}, \mathrm{C}-\mathrm{H}_{\text {phenyl }}\right), 7.51-7.60(\mathrm{~m}, 2 \mathrm{H}, \mathrm{C} 7-\mathrm{H}, \mathrm{C}-\mathrm{H}$ phenyl), 7.67 (t, $1 \mathrm{H}, J 7.43 \mathrm{~Hz}, \mathrm{C} 8-$ $\mathrm{H}), 7.88(\mathrm{~d}, 1 \mathrm{H}, J 9.80 \mathrm{~Hz}, \mathrm{C}-\mathrm{H}$ phenyl) 7.91 (dd, J 8.80, $1.20 \mathrm{~Hz}, 1 \mathrm{H}, \mathrm{C}-\mathrm{H}$ phenyl), $8.18(\mathrm{~d}, 1 \mathrm{H}, J 7.43 \mathrm{~Hz}, \mathrm{C} 10-\mathrm{H}), 9.53$ $\left(\mathrm{s}, 1 \mathrm{H}, \mathrm{N}-\mathrm{H}\right.$ exchangeable in $\left.\mathrm{D}_{2} \mathrm{O}\right), 13.79\left(\mathrm{~s}, 1 \mathrm{H}, \mathrm{C} 4-\mathrm{OH}\right.$ exchangeable in $\left.\mathrm{D}_{2} \mathrm{O}\right),{ }^{13} \mathrm{C} \mathrm{NMR}\left(101 \mathrm{MHz}, \mathrm{DMSO}-d_{6}\right)$ $\delta_{c}: 13.7$ (s, C4 $), 20.1$ (s, C3'), 29.5 (s, C2 '), 42.4 (s, C1'), 100.0 (s, C3), 101.6 (s, C4a), 113.2 (s, C7), 115.1 (s, C10a), 115. 9 (s, $\left.C_{\text {phenyl }}\right), 116.2$ (s, $\left.C_{\text {phenyl }}\right), 124.1$ (s, C10), 124.8 (s, C9), 124.8 (s, C phenyl), 124.8 (s, $\left.C_{\text {phenyl }}\right), 132.6$ (s, C8), 133.9 (s, C $C_{\text {phenyl) }} 134.1$ (s, C $C_{\text {phenyl) }} 137.9$ (s, C6a), 157.0 (s, C10b), 159.3 (s, C4), 159.6 (s, C2), 162.5 (s, C5), 162.9 (s, C=O benzoyl). ESI-MS m/z: $423.2240[\mathrm{M}+\mathrm{H}]^{+}, 445.1098[\mathrm{M}+\mathrm{Na}]^{+}, 446.1020[\mathrm{M}+\mathrm{H}+\mathrm{Na}]^{2+}, 867.3456$ $[2 \mathrm{M}+\mathrm{Na}]^{+}$. Anal. Calcd for $\mathrm{C}_{23} \mathrm{H}_{19} \mathrm{FN}_{2} \mathrm{O}_{5}$ (422.42): $\mathrm{C}, 65.40 ; \mathrm{H}, 4.53 ; \mathrm{N}, 6.63$. Found $\mathrm{C}, 65.15 ; \mathrm{H}, 4.43 ; \mathrm{N}, 6.61 \%$.

$\mathbf{N}$-(6-n-Butyl-4-hydroxy-2,5-dioxo-5,6-dihydro-2H-pyrano[3,2-c]quinolin-3-yl)-4-fluorobenzamide (14b). Pale yellow powder (3.5g, 87\%), mp > $300{ }^{\circ} \mathrm{C}$. IR $\left(\mathrm{KBr}, \mathrm{cm}^{-1}\right): 3432(\mathrm{O}-\mathrm{H}), 3266(\mathrm{NH}), 3064\left(\mathrm{CH}_{\text {aromatic }}\right), 2959,2930$, 2859 ( $\left.\mathrm{CH}_{\text {aliphatic. }}\right), 1748$ ( $\left.\mathrm{C}=\mathrm{O}_{\text {a-pyrone }}\right), 1677\left(\mathrm{C}=\mathrm{O}_{\text {quinolone }}\right), 1639$ ( $\left.\mathrm{C}=\mathrm{O}_{\text {arylcarbonyl }}\right)$ and 1573 (C=Caromatic). ${ }^{1} \mathrm{H} \mathrm{NMR}(400$ $\left.\mathrm{MHz}, \mathrm{DMSO}-d_{6}\right) \delta_{H}: 0.92\left(\mathrm{t}, J 8.00 \mathrm{~Hz}, 3 \mathrm{H}, \mathrm{C} 4^{\prime}\right), 1.35-1.46\left(\mathrm{~m}, 2 \mathrm{H}, \mathrm{C} 3^{\prime}\right), 1.60-1.70\left(\mathrm{~m}, 2 \mathrm{H}, \mathrm{C} 2^{\prime}\right), 4.34(\mathrm{t}, J 8.00$ $\left.\mathrm{Hz}, 2 \mathrm{H}, \mathrm{C} 1^{\prime}\right), 7.34\left(\mathrm{t}, J 8.00 \mathrm{~Hz}, 2 \mathrm{H}, \mathrm{C}-\mathrm{H}_{\text {phenyl }}\right), 7.53(\mathrm{t}, 1 \mathrm{H}, \mathrm{J} 7.24 \mathrm{~Hz}, \mathrm{C} 9-\mathrm{H}), 7.83-7.93(\mathrm{~m}, 2 \mathrm{H}, \mathrm{C} 7-\mathrm{H}, \mathrm{C}-\mathrm{H}$ phenyl), $8.00-8.08\left(\mathrm{~m}, 2 \mathrm{H}, \mathrm{C} 8-\mathrm{H}, \mathrm{C}-\mathrm{H}_{\text {phenyl) }}\right), 7.88$ (d, $1 \mathrm{H}, \mathrm{J} 9.80 \mathrm{~Hz}, \mathrm{C}-\mathrm{H}$ phenyl) 7.91 (dd, J 8.80, $1.20 \mathrm{~Hz}, 1 \mathrm{H}, \mathrm{C}-\mathrm{H}_{\text {phenyl }}$ ), $8.15(\mathrm{dd}, J$ 8.02, $1.37 \mathrm{~Hz}, 1 \mathrm{H}, \mathrm{C} 10-\mathrm{H}), 9.70\left(\mathrm{~s}, 1 \mathrm{H}, \mathrm{N}-\mathrm{H}\right.$ exchangeable in $\left.\mathrm{D}_{2} \mathrm{O}\right), 13.75(\mathrm{~s}, 1 \mathrm{H}, \mathrm{C} 4-\mathrm{OH}$ exchangeable

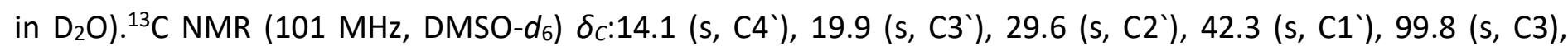
102.1 (s, C4a), 113.6 (s, C7 ), 115.7 (s, C10a ), 115.9 (s, C phenyl), 116.7 (s, C phenyl ), 124.2 (s, C10 ), 124.7 (s, C9), 130.8 (s, C8), 130.9 (s, $\left.C_{\text {phenyl }}\right), 130.9$ (s, $\left.C_{\text {phenyl }}\right), 134.7$ (s, $\left.C_{\text {phenyl }}\right), 138.1$ (s, C6a ), 157.2 (s, C10b ), 159.3 (s, C4), 163.1 (s, C2), 163.3 (s, C5), 163.5 (s, C=O benzoyl), 164.8 (s, C-F). ESI-MS m/z: $423.2758[\mathrm{M}+\mathrm{H}]^{+}, 445.0197$ $[\mathrm{M}+\mathrm{Na}]^{+}, 446.1732[\mathrm{M}+\mathrm{H}+\mathrm{Na}]^{2+}, 867.3784[2 \mathrm{M}+\mathrm{Na}]^{+}$. Anal. Calcd for $\mathrm{C}_{23} \mathrm{H}_{19} \mathrm{FN}_{2} \mathrm{O}_{5}$ (422.42): $\mathrm{C}, 65.40 ; \mathrm{H}, 4.53 ; \mathrm{N}$, 6.63. Found C, 65.35; H, 4.39; N, 6.60\%. 
N-(6-n-Butyl-4-hydroxy-2,5-dioxo-5,6-dihydro-2H-pyrano[3,2-c]quinolin-3-yl)-4-(trifluoromethoxy)

benzamide (14c). Yellow powder (3 g, 64\%), mp 255-257 ${ }^{\circ} \mathrm{C} . \mathrm{IR}\left(\mathrm{KBr}, \mathrm{cm}^{-1}\right): 3447(\mathrm{O}-\mathrm{H}), 3292(\mathrm{NH}), 3130$ ( $\left.\mathrm{CH}_{\text {aromatic }}\right), 2958,2932,2876$ ( $\left.\mathrm{CH}_{\text {aliphatic. }}\right), 1728$ ( $\left.\mathrm{C}=\mathrm{O}_{\text {a-pyrone }}\right), 1679\left(\mathrm{C}=\mathrm{O}_{\text {quinolone }}\right), 1613\left(\mathrm{C}=\mathrm{O}_{\text {arylcarbonyl }}\right)$ and 1576 (C=Caromatic). ${ }^{1} \mathrm{H}$ NMR $\left(400 \mathrm{MHz}, \mathrm{DMSO}-d_{6}\right) \delta_{H}: 0.91\left(\mathrm{t}, 1 \mathrm{H}, J 7.24 \mathrm{~Hz}, 3 \mathrm{H}, \mathrm{C} 4{ }^{\prime}\right), 1.35-1.46\left(\mathrm{~m}, 2 \mathrm{H}, \mathrm{C}^{\prime}\right), 1.59-$ $1.69\left(\mathrm{~m}, 2 \mathrm{H}, \mathrm{C} 2^{\prime}\right), 4.33\left(\mathrm{t}, 2 \mathrm{H}, \mathrm{J} 8.00 \mathrm{~Hz}, \mathrm{C} 1^{\prime}\right), 7.47-7.54(\mathrm{~m}, 3 \mathrm{H}) 7.81-7.85(\mathrm{~m}, 1 \mathrm{H}), 7.86-7.91(\mathrm{~m}, 1 \mathrm{H}), 8.07$ - $8.14(\mathrm{~m}, 3 \mathrm{H}),\left(\mathrm{s}, \mathrm{N}-\mathrm{H}\right.$ exchangeable in $\left.\mathrm{D}_{2} \mathrm{O}\right), 13.75\left(\mathrm{~s}, \mathrm{C} 4-\mathrm{OH}\right.$ exchangeable in $\left.\mathrm{D}_{2} \mathrm{O}\right) .{ }^{13} \mathrm{C} \mathrm{NMR}(101 \mathrm{MHz}$, DMSO-d $\left.d_{6}\right) \delta_{c}: 14.1\left(\mathrm{~s}, \mathrm{C} 4^{\prime}\right), 19.9$ (s, C3'), 29.6 (s, C2 '), 42.3 (s, C1 '), 99.8 (s, C3), 101.9 (s, C4a), 113.5 (s, C7), 116.7 (s, C10a ), 119.1 (s, OCF 3 ), 121.1 (s, C phenyl) $_{1} 121.6$ (s, C

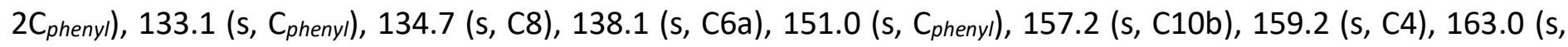
C2), 163.5 (s, C5), 164.7 (s, C=O benzoyl). ESI-MS m/z: $489.2773[\mathrm{M}+\mathrm{H}]^{+}, 511.2984[\mathrm{M}+\mathrm{Na}]^{+}, 977.2922[2 \mathrm{M}+\mathrm{H}]^{+}$, 999.3752 [2M+Na] $]^{+}$. Anal. Calcd for $\mathrm{C}_{24} \mathrm{H}_{19} \mathrm{~F}_{3} \mathrm{~N}_{2} \mathrm{O}_{6}$ (488.42): C, 59.02; H, 3.92; N, 5.74. Found C, 58.97; $\mathrm{H}, 3.89$; N, $5.67 \%$.

General procedure for preparation of oxazolopyranoquinolinone derivatives 15a-c. A mixture of compound 14a-c $(10 \mathrm{mmol})$ and glacial $\mathrm{AcOH}(15 \mathrm{~mL})$ with $\mathrm{Ac}_{2} \mathrm{O}(10 \mathrm{~mL})$ was heated under reflux for $12 \mathrm{~h}$. During this time the targeted compounds $\mathbf{1 5 a - c}$ were precipitated. After completion of the reaction (confirmed by TLC), the reaction mixture was cooled to rt. The obtained solid was filtered off, washed with EtOH $(3 \times 10 \mathrm{~mL})$ and crystallized from $\mathrm{AcOH}$ to give the corresponding compound 15.

5-n-Butyl-2-(2-fluorophenyl)-4H-oxazolo[5',4':4,5]pyrano[3,2-c]quinoline-4,11(5H)-dione (15a). White powder (3.4 g, 84\%), mp 260-262 ${ }^{\circ} \mathrm{C} . \mathrm{IR}\left(\mathrm{KBr}, \mathrm{cm}^{-1}\right): 3077\left(\mathrm{CH}_{\text {aromatic }}\right), 2952,2935,2869\left(\mathrm{CH}_{\text {aliphatic. }}\right), 1771\left(\mathrm{C}=\mathrm{O}_{\alpha-}\right.$

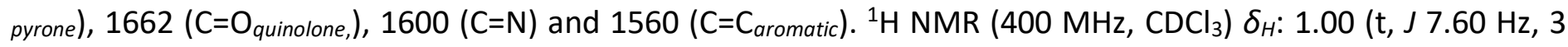
$\left.\mathrm{H}, \mathrm{C}^{\prime}\right)$ ) 1.46-1.58 (m, $\left.2 \mathrm{H}, \mathrm{C}^{\prime}\right), 1.71-1.85\left(\mathrm{~m}, 2 \mathrm{H}, \mathrm{C} 2^{\prime}\right), 4.37$ (t, J 8.00 Hz, $\left.2 \mathrm{H}, \mathrm{C} 1^{\prime}\right), 7.2-7.27\left(\mathrm{~m}, 1 \mathrm{H}, \mathrm{C}-\mathrm{H}_{\text {phenyl }}\right)$, 7.28 - $7.33\left(\mathrm{~m}, 1 \mathrm{H}, \mathrm{C}-\mathrm{H}_{\text {phenyl }}\right), 7.37(\mathrm{t}, 1 \mathrm{H}, J 7.63 \mathrm{~Hz}, \mathrm{C} 9-\mathrm{H}), 7.44(\mathrm{~d}, 1 \mathrm{H}, J 8.61 \mathrm{~Hz}, \mathrm{C} 7-\mathrm{H}, \mathrm{)}), 7.51-7.58(\mathrm{~m}, 1 \mathrm{H}, \mathrm{C}-$ $\left.\mathrm{H}_{\text {phenyl }}\right), 7.68-7.74(\mathrm{~m}, 1 \mathrm{H}, \mathrm{C} 8-\mathrm{H}), 8.28\left(\mathrm{dd}, 1 \mathrm{H}, \mathrm{J} 7.60,1.60 \mathrm{~Hz}, \mathrm{C}-\mathrm{H}_{\text {phenyl }}\right), 8.32(\mathrm{dd}, 1 \mathrm{H}, \mathrm{J} 8.02,1.76 \mathrm{~Hz}, \mathrm{C} 10-\mathrm{H})$, ${ }^{13} \mathrm{C}$ NMR (101 MHz, CDCl 3 ) $\delta_{c}: 13.8$ (s, C4) 20.2 (s, C3'), 29.5 (s, C2 '), 42.6 (s, C1'), 100.8 (s, C4a), 113.4 (s,

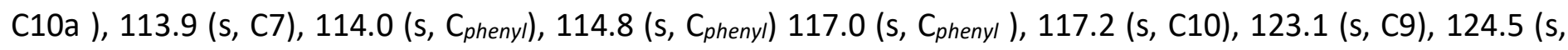

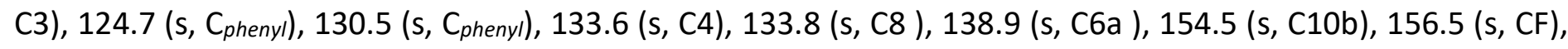
157.3 (s, C2 oxazol), 159.3 (s, C2), 161.9 (s, C5). ESI-MS m/z: $405.1330[\mathrm{M}+\mathrm{H}]^{+}, 406.1340[\mathrm{M}+2 \mathrm{H}]^{2+}, 831.3452$ [2M+Na] $]^{+}$. Anal. Calcd for $\mathrm{C}_{23} \mathrm{H}_{17} \mathrm{FN}_{2} \mathrm{O}_{4}$ (404.40): C, 68.31; $\mathrm{H}, 4.24 ; \mathrm{N}, 6.93$. Found $\mathrm{C}, 68.28 ; \mathrm{H}, 4.09 ; \mathrm{N}, 6.88 \%$.

5-n-Butyl-2-(4-fluorophenyl)-4H-oxazolo[5',4':4,5]pyrano[3,2-c]quinoline-4,11(5H)-dione (15b). White powder (3.7 g, 91\%), mp 236-238 ${ }^{\circ} \mathrm{C}$. IR $\left(\mathrm{KBr}, \mathrm{cm}^{-1}\right): 3080\left(\mathrm{CH}_{\text {aromatic }}\right), 2955,2932,2869\left(\mathrm{CH}_{\text {aliphatic. }}\right), 1788(\mathrm{C}=\mathrm{O} \alpha$ -

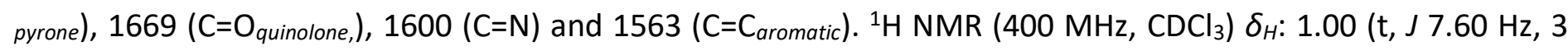
H, C4 $\left.{ }^{\prime}\right), 1.47$ - $1.57\left(\mathrm{~m}, 2 \mathrm{H}, \mathrm{C}^{\prime}\right), 1.73-1.82\left(\mathrm{~m}, 2 \mathrm{H}, \mathrm{C} 2^{\prime}\right), 4.36\left(\mathrm{t}, J 7.80 \mathrm{~Hz}, 2 \mathrm{H}, \mathrm{C}^{\prime}\right), 7.20(\mathrm{t}, 1 \mathrm{H}, J 8.80 \mathrm{~Hz}, \mathrm{C} 9-$ H), 7.36 (dd, $1 \mathrm{H}, J 7.70,1.20 \mathrm{~Hz}, \mathrm{C}-\mathrm{H}_{\text {phenyl) }}, 7.40$ (dd, $1 \mathrm{H}, J 6.80,0.80 \mathrm{~Hz}, \mathrm{C}-\mathrm{H}_{\text {phenyl) }}, 7.44(\mathrm{~d}, 1 \mathrm{H}, J 8.61 \mathrm{~Hz}, \mathrm{C} 7-$ H), 7.46 (d, $1 \mathrm{H}, J 5.87 \mathrm{~Hz}, \mathrm{C}-\mathrm{H}$ phenyl), 7.72 (t, $1 \mathrm{H}, J 7.60 \mathrm{~Hz}, \mathrm{C} 8-\mathrm{H}), 8.31$ (dd, $1 \mathrm{H}, J$ 5.20, $2.00 \mathrm{~Hz}, \mathrm{C}-\mathrm{H}_{\text {phenyl) }}, 8.34$

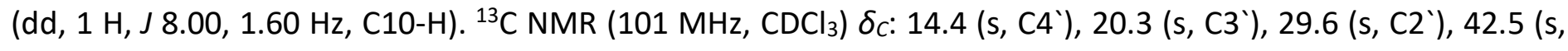
C1) 100.9 (s, C4a), 113.5 (s, C10a ), 114.8 (s, C7 ), 116.28 (s, Cphenyl), 116.5 (s, Cphenyl), 123.1 (s, C3), 124.7 (s, C10 ), 125.9 (s, C9), 130.1 (s, C8), 133.4 (s, C C4), 156.0 (s, C10b), 156.6 (s, C2 oxazol), 156.9 (s, C2), 163.8 (s, C5), 164.7 (s, CF). ESI-MS m/z: 405.2520 [M+H] , 831.3457 [2M+Na] $]^{+}$. Anal. Calcd for $\mathrm{C}_{23} \mathrm{H}_{17} \mathrm{FN}_{2} \mathrm{O}_{4}$ (404.40): C, 68.31; H, 4.24; N, 6.93. Found C, 68.22; $\mathrm{H}, 4.15 ; \mathrm{N}$, $6.70 \%$.

5-n-Butyl-2-(4-(trifluoromethoxy)phenyl)-4H-oxazolo[5',4':4,5]pyrano[3,2-c]quinoline-4,11(5H)-dione. (15c). White powder $(4.4 \mathrm{~g}, 94 \%), \mathrm{mp} 245-247^{\circ} \mathrm{C} . \mathrm{IR}\left(\mathrm{KBr}, \mathrm{cm}^{-1}\right): 3084\left(\mathrm{CH}_{\text {aromatic }}\right), 2958,2932,2879\left(\mathrm{CH}_{\text {aliphatic. }}\right), 1755$ ( $\left.\mathrm{C}=\mathrm{O}_{\alpha \text {-pyrone }}\right), 1666\left(\mathrm{C}=\mathrm{O}_{\text {quinolone }}\right), 1596(\mathrm{C}=\mathrm{N})$ and $1560\left(\mathrm{C}=\mathrm{C}_{\text {aromatic }}\right) .{ }^{1} \mathrm{H} \mathrm{NMR}\left(400 \mathrm{MHz}, \mathrm{CDCl}_{3}\right) \delta_{H}: 1.02(\mathrm{t}, J 7.43$

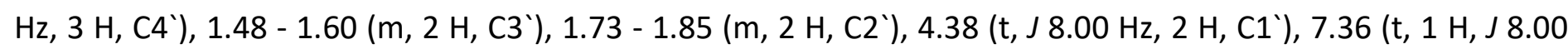


$\mathrm{Hz}, \mathrm{C} 9-\mathrm{H}), 7.40$ (d, J $7.20 \mathrm{~Hz}, 2 \mathrm{H}, \mathrm{C}-\mathrm{H}_{\text {phenyl) }}, 7.46$ (d, $\left.1 \mathrm{H}, J 8.61 \mathrm{~Hz}, \mathrm{C} 7-\mathrm{H}\right), 7.73(\mathrm{t}, 1 \mathrm{H}, J 7.60 \mathrm{~Hz}, \mathrm{C} 8-\mathrm{H}), 8.34(\mathrm{dd}$, J 7.6, $1.2 \mathrm{~Hz}, 2 \mathrm{H}, \mathrm{C}-\mathrm{H}_{\text {phenyl) }}, 8.37$ (dd, $1 \mathrm{H}, J$, 8.00, $\left.1.6 \mathrm{~Hz}, \mathrm{C} 10-\mathrm{H}\right) .{ }^{13} \mathrm{C} \mathrm{NMR}\left(101 \mathrm{MHz}, \mathrm{CDCl}_{3}\right) \delta_{\mathrm{C}}: 13.8$ (s, C4), 20.3 (s, C3'), 29.5 (s, C2 ) , 42.6 (s, C1'), 100.8 (s, C4a ), 113.4 (s, C10a ), 114.9 (s, C7 ), 121.1 (s, C phenyl), 123.2 (s, C10), 123.9 (s, C9), 124.7 (s, C phenyl), 125.9 (s, C3), 129.5 (s, C phenyl), 133.6 (s, C-F3), 138.9 (s, C6a), 151.9 (s, C4), 154.7 (s, $\left.\mathrm{C}_{\text {phenyl) }}\right) 155.9$ (s, C10b), 156.6 (s, $\left.\mathrm{C}_{\text {phenyl }}\right), 157.3$ (s, C2 oxazol), 160.8 (s, C2 ), 162.8 (s, C5). ESI-MS m/z: $471.1[\mathrm{M}+\mathrm{H}]^{+}, 493.2[\mathrm{M}+\mathrm{Na}]^{+}$. Anal. Calcd for $\mathrm{C}_{24} \mathrm{H}_{17} \mathrm{~F}_{3} \mathrm{~N}_{2} \mathrm{O}_{5}$ (470.41): C, 61.28; H, 3.64; N, 5.96. Found C, 61.20; $H, 3.57 ; \mathrm{N}, 5.89 \%$.

\section{References}

1. Anniyappan, M.; Muralidhran, D.; Perumal, P. T. Tetrahedron Lett. 2003, 44, 3653. https://doi.org/10.1016/S0040-4039(03)00707-X

2. Ravindranath, N.; Ramesh, C.; Reddy, M. R.; Das, B. Chem. Lett. 2003, 32, 222. https://doi.org/10.1246/cl.2003.222

3. Arasakumar, T.; Mathusalini, S.; Gopalan, S.; Shyamsivappan, S.; Ata, A.; Mohan, P. S. Bioorg. Med. Chem. Lett. 2017, 1538. https://doi.org/10.1016/j.bmcl.2017.02.042

4. Tomassoli, I.; Herlem, G.; Picaud, F.; Benchekroun, M.; Bautista-Aguilera, O. M.; Luzet, V.; Jimeno, M.-L.; Gharbi, T.; Refouvelet, B.; Ismaili, L. Monatsh. Chem. 2016, 147, 1069. https://doi.org/10.1007/s00706-016-1660-7

5. Ramesh, E.; Manian, R. S.; Raghunathan, R.; Sainath, S.; Raghunathan, M. Bioorg. Med. Chem. 2009, 17, 660.

https://doi.org/10.1016/j.bmc.2008.11.058

6. Anniyappan, M.; Muralidhran, D.; Perumal, P. T. Tetrahedron Lett. 2003, 44, 3653. https://doi.org/10.1016/S0040-4039(03)00707-X

7. El-Agrody, A. M.; Abd-Rabboh, H. S. M.; Al-Ghamd A. M. Med. Chem. Res. 2013, 22, 1339. https://doi.org/10.1007/s00044-012-0142-7

8. Magedov I.V.; Manpadi M.; Ogasawara M. A.; Dhawan A.S. Rodelj S.;Van slambrouck S.; Steelant W. F. A.; Evdokimov N. M.; Uglinskii P. Y.;Elias E. M.; Knee E. J.; Tongwa P.; Antipin M. Y.; Kornienko A. J. Med. Chem. 2008, 51, 2561. https://doi.org/10.1021/im701499n

9. Giddens, A. C.; Boshoff, H. I. M.; Franzblau, S. G.; Barry, C. E.; Copp, B. R. Tetrahedron Lett. 2005, 46, 7355.

https://doi.org/10.1016/j.tetlet.2005.08.119

10. Zhao, Q.; Liu, S.; Li, Y.; Wang, Q.; J. Agric. Food Chem., 2009, 57, 2849. https://doi.org/10.1021/jf803632t

11. Chen, L.; Ren, Z.; Zhou. X.; Zeng, J.; Zou, J.; Li, Y. Appl. Microbiol. Biotechnol. 2016, 100, 857. https://doi.org/10.1007/s00253-015-7092-1

12. Gradillas, A.; Lopez, B.; Brana, Sancho, I.; Perez-Garkia, C.; Alguacil, L. F. Arzneim.-Forsch./Drug Res. 2005, 55, 725.

13. Zhang, F.; Chapman, K. T.; Schleif, W.A.; Olsen, D. B.; Stahlhut, M.; Rutkowski, C. A.; Kuo, L. C.; Jin, L.; Lin, J. H.; Emini, E. A.; Tata, J. R. Bioorg. Med. Chem. Lett. 2003, 13, 2573.

https://doi.org/10.1016/S0960-894X0300474-8 
14. Minhas, G. S.; Pilch, D. S.; Kerrigan, J. E.; La Voie, E. J.; Rice, J. E. Bioorg. Med. Chem. Lett. 2006, 16, 3891. https://doi.org/10.1016/j.bmcl.2006.05.038

15. Lipunova, G. N.; Nosova, E. V.; Charushin, V. N.; Chupakhin, O. N. J. Fluorine Chem. 2015, $175,84$. https://doi.org/10.1016/i.jfluchem.2015.03.011

16. Xia, Y.; Yang, Z. Y.; Xia, P.; Hackl, T.; Hamel, E.; Mauger, A.; Wu J. H.; Lee, K. H., J. Med. Chem. 2001, 44, 3932.

https://doi.org/10.1021/jm0101085

17. Al-Trawneh, S. A.; Zahra, J. A.; Kamal, M. R.; El-Abadelah, M. M.; Zani, F.; Incerti, M.; Cavazzoni, A.; Alfieri, R. R.; Petronini, P. G.; Vicini, P. Bioorg. Med. Chem. 2010, 18, 5873.

https://doi.org/10.1016/i.bmc.2010.06.098

18. Nosova, E. V.; Lipunova, G. N.; Charushin, V. N.; Chupakhin O. N. J. Fluorine Chem. 2010, 131, 1267. https://doi.org/10.1016/j.jfluchem.2010.09.007

19. Ibrahim, M. A.; Hassanin, H. M. Arkivoc 2013 (iv) 217.

https://doi.org/10.1016/j.jfluchem.2010.09.007

20. Hassanin, H. M. Arkivoc 2012, (vi), 384.

https://doi.org/10.1016/j.jfluchem.2010.09.007

21. Liu, Y.; Lu, Y.; Prashad, M.; Repič, O.; Blacklock, T. J. Adv. Synth. Catal. 2005, 347, 217. https://doi.org/10.1002/adsc.200404236

22. Sadavarte, V. S.; Swami, S. S.; Desai, D. G. Synth. Commun. 1998, 28, 1139. https://doi.org/10.1080/00397919808005954

23. Jampilek, J.; Musiol, R.; Pesko, M.; Kralova, K.; Vejsova, M.; Carroll, J.; Coffey, A.; Finster, J.; Tabak, D.; Niedbala, H.; Kozik, V.; Polanski, J.; Csollei, J.; Dohnal, J. Molecules, 2009, 14, 1145. https://doi.org/10.3390/molecules14031145

24. Kappe, T.; Aigner, R.; Hohengassner, P.; Stadlbauer, W. J. Prakt. Chem. 1994, 336, 596. https://doi.org/10.1002/prac.19943360707 